\title{
A Fundamental Climate Data Record of SMMR, SSM/I, and SSMIS brightness temperatures
}

\author{
Karsten Fennig $^{1}$, Marc Schröder ${ }^{1}$, Axel Andersson ${ }^{2}$, and Rainer Hollmann ${ }^{1}$ \\ ${ }^{1}$ Deutscher Wetterdienst, Offenbach, Germany \\ ${ }^{2}$ Deutscher Wetterdienst, Hamburg, Germany \\ Correspondence: Karsten Fennig (karsten.fennig@dwd.de)
}

Received: 21 August 2019 - Discussion started: 18 September 2019

Revised: 24 January 2020 - Accepted: 1 February 2020 - Published: 20 March 2020

\begin{abstract}
The Fundamental Climate Data Record (FCDR) of Microwave Imager Radiances from the Satellite Application Facility on Climate Monitoring (CM SAF) comprises inter-calibrated and homogenized brightness temperatures from the Scanning Multichannel Microwave Radiometer (SMMR), the Special Sensor Microwave/Imager (SSM/I), and the Special Sensor Microwave Imager/Sounder SSMIS radiometers. It covers the time period from October 1978 to December 2015 including all available data from the SMMR radiometer aboard Nimbus-7 and all SSM/I and SSMIS radiometers aboard the Defense Meteorological Satellite Program (DMSP) platforms. SMMR, SSM/I, and SSMIS data are used for a variety of applications, such as analyses of the hydrological cycle, remote sensing of sea ice, or as input into reanalysis projects. The improved homogenization and inter-calibration procedure ensures the long-term stability of the FCDR for climate-related applications. All available raw data records from different sources have been reprocessed to a common standard, starting with the calibration of the raw Earth counts, to ensure a completely homogenized data record. The data processing accounts for several known issues with the instruments and corrects calibration anomalies due to along-scan inhomogeneity, moonlight intrusions, sunlight intrusions, and emissive reflector. Corrections for SMMR are limited because the SMMR raw data records were not available. Furthermore, the inter-calibration model incorporates a scene dependent inter-satellite bias correction and a non-linearity correction in the instrument calibration. The data files contain all available original sensor data (SMMR: Pathfinder level 1b) and metadata to provide a completely traceable climate data record. Inter-calibration and Earth incidence angle normalization offsets are available as additional layers within the data files in order to keep this information transparent to the users. The data record is complemented with noise-equivalent temperatures $(\mathrm{Ne} \Delta \mathrm{T})$, quality flags, surface types, and Earth incidence angles. The FCDR together with its full documentation, including evaluation results, is freely available at: https://doi.org/10.5676/EUM_SAF_CM/FCDR_MWI/V003 (Fennig et al., 2017).
\end{abstract}

1

\section{Introduction}

Data from space-borne microwave imagers and sounders such as the Scanning Multichannel Microwave Radiometer (SMMR), Special Sensor Microwave/Imager (SSM/I), and Special Sensor Microwave Imager/Sounder (SSMIS) are used for a variety of applications, such as analyses of the hydrological cycle (precipitation and evaporation) and related atmospheric and surface parameters (Andersson et al., 2011), as well as remote sensing of sea ice (Lavergne et al., 2019), soil moisture (Dorigo et al., 2017), and land surface temperatures (Prigent et al., 2016). Carefully calibrated and homogenized radiance data records are a fundamental prerequisite for climate analysis, climate monitoring, and reanalysis (Dee et al., 2011; Hersbach et al., 2018). Several National Meteorological and Hydrological Service (NMHS) centres assimilate microwave radiances directly. Forecast and reanalysis can thus benefit from a Fundamental Climate Data Record (FCDR) of brightness temperatures $\left(T_{\mathrm{B}}\right)$ (Poli et al., 2013). An FCDR is a well-characterized, long-term data record, 
compiled from a series of similar, quality-controlled, and calibrated series of similar instruments. Each individual instrument and its successor must have sufficient calibration and overlap to enable a successful inter-calibration of the measurements from all instruments. This way the generation of products that are accurate and stable to support climate analysis is allowed (GCOS-200, 2017). FCDRs are a prerequisite for the generation of Thematic Climate Data Records (TCDRs). The highest possible TCDR quality can only be achieved by harmonizing the satellite observations in radiance space, in turn increasing the product's value for users.

The aim of the data record, presented in this paper, is to provide such an FCDR of observed brightness temperatures for each individual instrument along with separate inter-calibration offset values to homogenize the observations across all different sensors.

The predecessors of this data record and the data processor suite were originally developed at the Max Planck Institute for Meteorology (MPI-M) and the University of Hamburg (UHH) for the Hamburg Ocean Atmosphere Parameters and Fluxes from Satellite Data (HOAPS) climatology. HOAPS is a compilation of climate data records for analysing the water cycle components over the global oceans derived from satellite observation (Andersson et al., 2011). The main satellite instrument employed to retrieve the geophysical parameters was the SSM/I, and much work has been invested to process and carefully homogenize all SSM/I instruments aboard the Defense Meteorological Satellite Program (DMSP) platforms F08, F10, F11, F13, F14, and F15 (Andersson et al., 2010).

The HOAPS processing suite was then transferred to the Satellite Application Facility on Climate Monitoring (CM SAF) in a research-to-operation action in order to provide a sustained environment to process climate data records, which is one of the main tasks of CM SAF (Schulz et al., 2009). The operational processing and reprocessing of the FCDRs and TCDRs, as well as the provision to the research community, are maintained and coordinated by the CM SAF.

With the transfer of the HOAPS data record to CM SAF, a high demand for an FCDR was identified. However, at that time no FCDR providing all SSM/I and SSMIS data and metadata was freely available. Thus, it was decided to utilize the inter-calibration work from the HOAPS processing suite to compile a new FCDR, meeting all requirements. The first release of the CM SAF FCDR (Fennig et al., 2013) focused on the SSM/I series, covering the time period from 1987 to the end of 2008. In order to continue the HOAPS TCDRs beyond 2008 it was also necessary to extend the underlying FCDR of microwave brightness temperatures with the SSMIS sensor family aboard the DMSP platforms F16, F17, and F18. This was accomplished with the second release of the CM SAF FCDR (Fennig et al., 2015). This combined FCDR of SSM/I and SSMIS brightness temperatures provides a consistent FCDR from 1987 to 2013.
Following requests from users of the FCDR, the third release (Fennig et al., 2017) focused on the extension of the microwave brightness temperature data record to the earlier time period from 1978 to 1987 with observations from SMMR aboard Nimbus-7. However, this turned out to be a challenging task, as it has not been possible to get a hold of the original raw instrument data records. Instead, the Nimbus-7 SMMR Pathfinder level 1B Brightness Temperatures data record (Njoku, 2003), available from the National Snow and Ice Data Center (NSIDC), has been used to generate this FCDR. This work was also a part of data rescue projects aiming to provide FCDRs of historical satellite data to enhance the data coverage for reanalysis projects in the 1960s and 1970s (Poli et al., 2017).

This is not the only FCDR of passive microwave radiometry. Within the Climate Data Record Program of the National Oceanic and Atmospheric Administration (NOAA), two FCDRs have been developed: one from the Colorado State University (CSU) (Kummerow et al., 2013) and a second one from Remote Sensing Systems (RSS) (Wentz et al., 2013). Both use different methods to inter-calibrate the sensors and cover different platforms and time periods. Within the Global Precipitation Measurement (GPM) project, a further data record has been developed with the aim to utilize all available microwave imagers to provide high-quality global precipitation estimates (Berg et al., 2018), which is based on the FCDR from CSU (Kummerow et al., 2013) but covers more platforms.

This paper is structured as follows. A short description of the satellite instruments is given in Sect. 2. In Sect. 3 the data processing is explained. This is followed by a description of how the brightness temperatures are derived in Sect. 4 and how the instruments are inter-calibrated in Sect. 5. Uncertainties are discussed in Sect. 6. In Sect. 7 the FCDR is evaluated, and finally the conclusions are provided in Sect. 9. The instrument-related specifications are always presented in a logical sequence starting with SSM/I as the most important contributor to the FCDR, followed by SSMIS and finally SMMR.

\section{Instrumentation and data provenance}

\subsection{The SSM/I instrument}

SSM/I sensors have been operated aboard the DMSP satellites as part of the global satellite observing system since 1987. Up to three satellites have been in orbit simultaneously. An extensive description of the instrument and satellite characteristics has been published by Hollinger et al. (1987) and Wentz (1991). A short summary of the information is given here.

The DMSP satellites operate in a near-circular, sunsynchronous orbit, with an inclination of $98.8^{\circ}$ at an approximate altitude of $860 \mathrm{~km}$. Each day, 14.1 orbits with a period of about 102 min are performed. The Earth's surface is sam- 
pled with a conical scan at a constant antenna boresight angle of $45^{\circ}$ over an angular sector of $102.4^{\circ}$ resulting in a $1400 \mathrm{~km}$ wide swath. A nearly complete coverage of the Earth by one $\mathrm{SSM} / \mathrm{I}$ instrument is achieved within 2 to $3 \mathrm{~d}$. Due to the orbit inclination and swath width, the regions poleward of $87.5^{\circ}$ are not covered.

Six SSM/I instruments have been successfully launched aboard the F08 (June 1987), F10 (December 1990), F11 (November 1991), F13 (March 1995), F14 (April 1997), and F15 (December 1999) spacecraft. All satellites have a local Equator crossing time between 05:00 and 10:00 for the descending node and between 17:00 and 22:00 for the ascending node. The F08 had a reversed orbit with the ascending node in the morning. Also, the Earth observing angular sector of the scan on this satellite is, differently from the others, centred to the aft of the sub-satellite track.

The SSM/I is a seven-channel total power radiometer measuring emitted microwave radiation at four frequency intervals centred at 19.35, 22.235, 37.0, and $85.5 \mathrm{GHz}$. All frequencies are sampled at horizontal (h-pol) and vertical polarization (v-pol), except for the $22.235 \mathrm{GHz}$ channel, which measures only vertically polarized radiation. Due to thermal problems, the instrument aboard the F08 had to be switched off in December 1987. When the instrument was reactivated, the data quality of the channels at $85.5 \mathrm{GHz}$ degraded quickly (Hollinger et al., 1990). Hence, the high-frequency channels of SSM/IF08 are regarded as defective from April 1998 onwards.

The size of an instantaneous field of view (footprint) is defined as the distance where the antenna power has dropped to half of its peak value. The diameters of this ellipse vary with frequency. They range from $69 \mathrm{~km}$ along the track of the spacecraft by $43 \mathrm{~km}$ across the track with a sampling frequency of $25 \mathrm{~km}$ for the $19 \mathrm{GHz}$ channel to $15 \mathrm{~km}$ by $13 \mathrm{~km}$ with $12.5 \mathrm{~km}$ sampling frequency for the $85 \mathrm{GHz}$ channel. The $85 \mathrm{GHz}$ channels are sampled for each rotation of the instrument with a resolution of 128 uniformly spaced pixels, while the remaining channels are sampled every other scan with a resolution of 64 pixels. A fixed cold-space reflector and a reference black-body warm load are used for continuous on-board two-point calibration.

The main data source for the SSM/I data is the National Environmental Satellite, Data, and the Information Service (NESDIS) temperature data record (TDR), available from NOAA's Comprehensive Large Array-data Stewardship System (CLASS). For data earlier than 1993, not covered by the NESDIS TDR, reformatted NetCDF TDR data files from the National Centers for Environmental Information (NCEI) (Semunegus et al., 2010) are used. These are based on SSM/I Antenna Temperature Tapes from RSS (Wentz, 1991) for this time period.

\subsection{The SSMIS instrument}

The SSMIS is the successor to the SSM/I. An extensive description of the instrument and satellite characteristics has been published by Kunkee et al. (2008b). A short summary of essential information is given in this section. The SSMIS instruments are operated, as SSM/I, aboard the DMSP satellites in an early-morning orbit, continuing the existing data record at the same overpass time. The SSMIS has a wider Earth viewing angular sector $\left(144^{\circ}\right)$ than the SSM/I, resulting in a $1700 \mathrm{~km}$ wide swath. While four SSMIS instruments have been successfully launched aboard the F16 (October 2003), F17 (November 2006), F18 (October 2009), and F19 (April 2014) spacecraft, only the first three provide a sufficient data coverage for climate applications. The F19 spacecraft failed about 2 years after launch.

The SSMIS integrates the imaging capabilities of the SSM/I sensor with the cross-track microwave sounder Special Sensor Microwave Temperature (SSM/T) and Special Sensor Microwave Humidity Sounder (SSM/T-2) into a single conically scanning 24-channel instrument. The SSM/Ilike frequencies are centred at 19.35, 22.235, 37.0, and $91.35 \mathrm{GHz}$. All frequencies are sampled at horizontal and vertical polarization, except for the $22.235 \mathrm{GHz}$ channel, which measures only vertically polarized radiation.

The footprint size varies from $74 \mathrm{~km}$ by $47 \mathrm{~km}$ with an along-scan sampling frequency of $25 \mathrm{~km}$ for the $19 \mathrm{GHz}$ channel to $15 \mathrm{~km}$ by $13 \mathrm{~km}$ with $12.5 \mathrm{~km}$ along-scan sampling frequency for the $91 \mathrm{GHz}$ channels. All channels are sampled for each rotation, resulting in an along-track sampling of $12.5 \mathrm{~km}$ with a resolution of 180 uniformly spaced pixels. The SSM/I-like channels are averaged on board the spacecraft. Two neighbouring observations are averaged, resulting in a resolution of 90 pixels. A fixed cold-space reflector and a reference black-body warm load are used for continuous on-board two-point calibration.

The data records used as input for SSMIS are the CSU SSMIS BASE Temperature Data Records (Kummerow et al., 2013) and the original level 1A TDR data files available from NOAA's CLASS. The BASE data record is a collection of the level 1A unprocessed antenna temperature data that has been written into single orbit granules and reformatted into netCDF-4. These files contain all of the information from the original source TDR files plus additional metadata and updated geolocation information.

\subsection{The SMMR instrument}

A detailed description of the SMMR is given in Gloersen and Barath (1977) and Madrid et al. (1978). Hence, only a summary of essential information is given here.

Two SMMR instruments were operated, one aboard Nimbus-7 and one aboard Seasat (Seafaring Satellite). While only about 3 months of data from the Seasat mission exist, 
SMMR on Nimbus-7 delivered a data record covering nearly 8 years from 25 October 1978 until 20 August 1987.

The Nimbus-7 spacecraft operated in a sun-synchronous orbit with an inclination of $99^{\circ}$ and an average altitude of $955 \mathrm{~km}$. This configuration results in an orbital period of about $104 \mathrm{~min}$ and provided approximately 14 orbits per day. The SMMR is mounted on the forward side of the spacecraft in the direction of flight. Due to power limitations the SMMR instrument operated most of the time on a $50 \%$ duty cycle: $1 \mathrm{~d}$ "on" followed by $1 \mathrm{~d}$ "off".

The SMMR is a 10-channel radiometer, measuring microwave radiation from the Earth's atmosphere and surface in five frequencies at vertical and horizontal polarization. Six radiometers were integrated in the instrument, fed by one multi-spectral feedhorn. While the four radiometers at the lower frequencies (from 6.6 to $21 \mathrm{GHz}$ ) measured alternating polarization each half-scan, the other two at $37 \mathrm{GHz}$ continuously measured vertical and horizontal polarization.

The instrument's antenna consists of an offset parabolic reflector and the multi-frequency feed assembly. The reflector is mounted at a nadir angle of $42^{\circ}$, which results in an average Earth incidence angle (EIA) of $50.3^{\circ}$. The antenna rotated within $\pm 25^{\circ}$, centred about the sub-satellite track. This results in a $780 \mathrm{~km}$ wide swath at the Earth's surface. The scan velocity varied sinusoidally, being fastest at a $0^{\circ}$ azimuthal scan angle and slowest at the scan edges. A complete scan is accomplished after $4.096 \mathrm{~s}$. Cold space is used as the cold reference target, viewed directly through one of three cold-sky calibration horns. The warm calibration target is at the instrument's ambient temperature. The footprint size varies from $148 \mathrm{~km}$ by $95 \mathrm{~km}$ at $6.6 \mathrm{GHz}$ to $27 \mathrm{~km}$ by $18 \mathrm{~km}$ at $37 \mathrm{GHz}$.

Acquisition of Nimbus-7 SMMR data commenced at midnight of 25 October 1978. The SMMR operated continuously during a 3-week checkout period from start-up until 16 November 1978, at which time it began alternate-day operation. This was the normal mode for most of its mission. Under normal operations the SMMR was turned on near midnight GMT (corresponding to a descending Equator crossing near $0^{\circ}$ longitude) and turned off at approximately the same time the following day, in a continuing sequence. A special operations period occurred from 3 April to 6 June 1986, during which the SMMR was switched on (for $\sim 30 \mathrm{~min}$ ) and off (for $\sim 75 \mathrm{~min}$ ) more frequently. The antenna scan mechanism was turned off permanently on 20 August 1987 for safety reasons, marking the end of the scanning SMMR data record (Njoku et al., 1998).

The data record used as input for the FCDR is the Nimbus-7 SMMR Pathfinder level 1B Brightness Temperatures data record, available from the Distributed Active Archive Center (DAAC) at the National Snow and Ice Data Center (NSIDC) (Njoku, 2003).

\section{Data processing}

Generally the data processing is split into a series of processing steps. A generalized data flow diagram is shown in Fig. 1. The required individual processing steps depend on the instrument series and are summarized first in this section to give a general overview of the processing sequence. The specific steps and corrections are then described in more detail in the following sections.

The data processing starts from the original Raw Data Records (RDRs), which are described in Sect. 2 for the individual instruments. First, the RDRs are filtered to remove duplicate and erroneous scan lines. The brightness temperatures are reverted to obtain the original measured counts and a new first-guess radiometric calibration is derived (SSM/I, SSMIS). Then the geolocation is performed (SSM/I, SSMIS) and a water-land surface type classification is made. Intermediate level 1a data files in a common data format are produced. These are used to detect sunlight and moonlight intrusion events in the SSMI(S) data records and to produce intrusion correction maps. In the next processing step the individual sensor corrections are applied before the final radiometric calibration is derived. If sensor issues have been identified, a specific correction must be applied. These correction methods are either taken from the literature and adapted accordingly or newly developed. The corrections are applied to correct along-scan non-uniformity, SSMI(S) moonlight intrusions, SSMI(S) sunlight intrusions, and SSMIS emission from the main reflector. The corrections are described in detail in Sect. 3.6. After applying the corrections, brightness temperatures and inter-calibration offsets are computed and the results are written to intermediate level $1 \mathrm{~b}$ data files. This intermediate product is used to compile daily sea ice masks for the SSM/I and SSMIS covered time period. In the last processing step the final surface type classification is performed, the EIA normalization offsets are computed, an antenna pattern matching is performed for the high-resolution channels, and a final quality control of all fields of view (FOVs) is applied.

The final FCDR data files are compiled in a standardized NetCDF (Network Common Data Form) format, applicable to all sensor types. The CM SAF FCDR data files are organized as daily collections of all observations from each sensor. All sensor-specific data available in the individual RDR are provided as well as quality control flags, EIAs, averaged brightness temperatures, EIA normalization offsets, and inter-sensor calibration offsets. All FCDR product files are swath based and built following the same design principles. The record dimension of the data files is the scan integer time measured in seconds since 1 January 1987 (1970 for SMMR). Each file contains all possible scans for 1 complete day regardless of their quality and status. Missing scans are marked as missing and all sensor data are set to undefined in this case. 
To account for the different sampling rate and alignment of the instrument feedhorns, each feedhorn is available as a separate logical data group. Each of the data groups provides a set of geolocation variables and can be used independently. Calibration data and spacecraft-related variables are also available in separate logical data groups. This flexible format design allows it to provide the different microwave conical scanning instrument data in one logical format.

Brightness temperatures, inter-sensor offsets, and EIA normalization offsets are archived as separate variables to allow the corrections to be added as required by the users. Adding EIA normalization offsets to the brightness temperatures results in a homogenized temperature data record where all observations are at a constant EIA. This is required when the retrieval algorithm does not account for the varying EIA. In order to apply the inter-calibration, the inter-calibration offsets must be added to the brightness temperatures. This step will bring all $T_{\mathrm{B}}$ values into alignment with the reference instrument.

A detailed description of the data files and usage is given in the corresponding instrument-specific product user manual (PUM), available from the DOI landing page at https:// doi.org/10.5676/EUM_SAF_CM/FCDR_MWI/V003 (Fennig et al., 2017).

\subsection{Overview}

\subsubsection{SSM/I}

A combined usage of different data sources is necessary for the SSM/I instruments, as they cover different time periods and have different coverage due to missing files, checksum errors, and corrupt data records. An analysis of SSM/I data from different sources (Ritchie et al., 1998), including NESDIS TDR and RSS data, showed no systematic differences between these data sets. Moreover, our own analysis showed that apart from the geolocation, which is different in the RSS files (Wentz, 1991), the same information is available in all data files.

To merge the scans from all different sources, unique MD5 hash values are computed from the calibration data. The MD5 algorithm is a cryptographic hash function that takes a block of data and returns a unique fixed size hash value that can be used as a fingerprint to identify a specific data block (Rivest, 1992). This MD5 hash value is used in the processing to find identical scan lines and to correctly merge all available data records to compile the FCDR files. During the first processing step antenna temperatures are reverted to Earth counts using the calibration coefficients provided in the data files. This must be done because the preprocessed TDR input data can contain erroneous calibration slopes and offsets caused by an averaging of the calibration data without quality control tests (Ritchie et al., 1998).

In the following processing step the spacecraft position and the geolocation are computed, and the water-land sur- face type is assigned to each field of view (FOV). A first raw data calibration is carried out to convert from Earth counts to antenna temperatures (TAs). From these intermediate level 1a data records, yearly monitoring files are compiled where the calibration and geolocation are sampled at fixed orbit angle positions. These files are analysed to identify periods where moonlight (cold view intrusion) or sunlight (hot view intrusion) affect the calibration in order to compile correction tables. They are then used to correct the cold and hot calibration counts. The final calibration coefficients are derived and the antenna temperatures are computed. An along-scan correction is applied to the TAs to account for a non-uniformity of the measured antenna temperatures along a scan line. The corrected antenna temperatures are then converted to brightness temperatures and the intersensor calibration offsets are computed.

Now the sea ice concentration is derived from this intermediate level $1 \mathrm{~b}$ data record for each FOV, and daily gridded ice masks are compiled (see Sect. 3.4). These maps are then used to assign the sea ice and sea ice margin surface types. The Earth incidence angle normalization offsets are computed for water-type FOVs and the high-resolution channels are averaged to provide additional information matching the antenna pattern at the lower resolution.

\subsubsection{SSMIS}

The SSMIS BASE data record files from CSU have been compiled from the original TDR and were reorganized into single orbit granules with duplicate scans mostly removed. Spacecraft position and velocity have been added by CSU based on North American Aerospace Defense Command (NORAD) orbital data.

Following the procedures developed for SSM/I, remaining duplicate scan lines in the SSMIS CSU BASE and the original TDR files are first screened and filtered using MD5 hash values of the calibration data. The SSMIS NORAD orbital data sets are not freely available. Therefore the spacecraft position in the CSU BASE data files is used to fit daily orbital data sets, which can then be used in the FOV geolocation procedure. The following processing steps are similar to the SSM/I processing. As an additional step for SSMIS, an emissive reflector correction is applied for $\mathrm{SSMIS}_{\mathrm{F} 16}$ and SSMIS $_{\mathrm{F} 17}$.

\subsubsection{SMMR}

The SMMR data record processing can not be performed starting from the actual observed Earth counts but has to start from the level 1B Pathfinder data record. Therefore the processing steps differ from SSMI(S) above.

A detailed description of the level 1B processing steps is given in Njoku et al. (1998) and summarized here for reference. The level 1B Pathfinder data record is a collection of $T_{\mathrm{B}}$ and instrument metadata, archived as single orbit gran- 


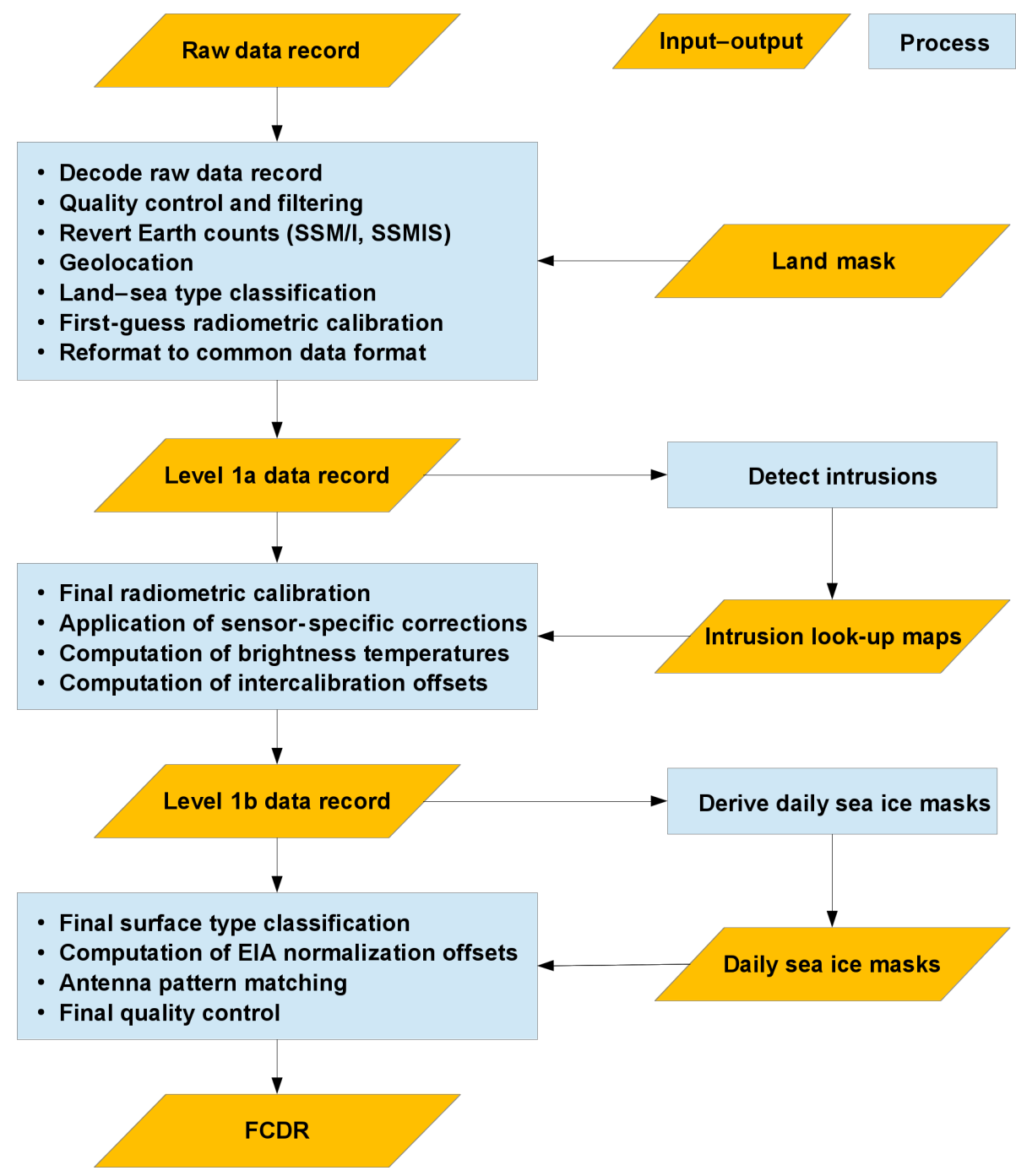

Figure 1. Generalized data flow diagram with the main processing steps to generate the CM SAF FCDR.

ules in Hierarchical Data Format (HDF). Input to the level 1B processor is level 1A antenna temperature tapes (TAT). The level $1 \mathrm{~B}$ data processing was performed in daily segments, and all algorithms used for running averages were initialized at the beginning of the day and also after data gaps longer than $1 \mathrm{~min}$. After reading and decoding, the radiometric calibration was carried out for each scan line. This was followed by spillover corrections, an absolute calibration correction, as well as corrections for long-term and short-term drifts. The resulting corrected antenna temperatures were then interpolated along and across scan lines to co-locate all observations to the $37 \mathrm{GHz}$ FOV locations. Finally the polarization mixing was corrected and a quality status flag was generated for each scan to indicate potential quality losses. This quality flag is also copied to the final FCDR data record for reference.

The CM SAF FCDR processing starts with a further filtering of this level 1B data record. Unique MD5 hash values are computed from the calibration data. All orbit granules for
$1 \mathrm{~d}$ are filtered using the MD5 hash values. The data record is also screened for erroneous geolocation, and valid scans are merged into daily SMMR FCDR NetCDF files. The scan times are only available rounded to full seconds. The original time fraction is needed for a correct navigation of the spacecraft position and is therefore estimated with a linear regression fit using all scans from $1 \mathrm{~d}$. The water-land surface type is assigned to each FOV using the same land-sea mask as for the SSMI(S) data records.

The radiometer noise-equivalent temperatures are estimated from recomputed calibration coefficients for each channel. An along-scan correction is applied to the $T_{\mathrm{B}}$ values at $37 \mathrm{GHz}$ to account for a non-uniformity of the measured $T_{\mathrm{B}}$ values along the scan line. Finally the inter-calibration offsets are computed, and quality flags and global metadata are assigned. 


\subsection{Geolocation}

\subsubsection{Geolocation for SSM/I and SSMIS}

The quality of the SSM/I geolocation has been examined by Poe and Conway (1990) and Colton and Poe (1999). The operational requirement of geolocating SSM/I data is defined by half the $3 \mathrm{~dB}$ beam diameter of the high-resolution $85 \mathrm{GHz}$ channels, which translates to a location error requirement of about $7 \mathrm{~km}$. The studies showed that the predicted spacecraft ephemeris in the data records caused an error of up to $15 \mathrm{~km}$. Since July 1989 the operational processing has changed and the new ephemeris error is typically less than $2-3 \mathrm{~km}$. To reduce the SSM/I FOV geolocation error below the requirement, a fixed set of spacecraft attitude corrections was determined (Colton and Poe, 1999). These attitude corrections were defined for each instrument. Colton and Poe (1999) finally conclude that the root-mean-square value of the geolocation error of the SSM/I FOV is less than $4 \mathrm{~km}$.

The quality of the SSMIS geolocation has been examined by Poe et al. (2008). This study showed geolocation errors in excess of $20-30 \mathrm{~km}$. An automated analysis methodology was developed during the F16 cal/val (calibration and validation) period. As the main sources of the errors, angular misalignments and timing problems have been identified. A set of fixed attitude corrections are found during the cal/val period for each SSMIS to reduce the geolocation error to less than $4-5 \mathrm{~km}$.

In order to provide a homogeneous and consistent geolocation in the CM SAF FCDR, the attitude corrections must be applied consistently throughout the entire time period. The geolocation data are therefore recalculated by CM SAF during the reprocessing using the latest available set of corrections. The spacecraft position is predicted with the Simplified General Perturbations model (SGP4) (Hoots and Roehrich, 1988). This model uses NORAD ephemeris data sets to predict position and velocity of Earth-orbiting objects. These orbital data are distributed in a two-line element (TLE) data format. The SSM/I element sets for the time period up to January 2000 are freely available and used until December 1999. SSMIS element sets are not freely available. In order to use the SGP4 model for the entire time period, ephemeris data from the raw data records have been used to find the orbit elements in a minimization fitting procedure for all SSM/I data files after 2000. For SSMIS, the spacecraft position, added in the BASE data files, is used to fit the orbit elements.

These fitted ephemeris data are determined for each day and then smoothed using a moving averaging window of $\pm 7 \mathrm{~d}$. A comparable method is used by Wentz (1991) to fit a simple orbit model. The accuracy is usually better than $1 \mathrm{~km}$ compared to original data and compared to time periods with available NORAD element sets. The spacecraft position is predicted for all scan start times and archived in the FCDR data files.
During the geolocation procedure the spacecraft position is then linearly interpolated to each pixel time in an Earthcentred inertial (ECI) coordinate frame by applying a rotation matrix. The rotation axis is found as the vector cross product between two consecutive spacecraft positions. The magnitude of the position vector is linearly interpolated. From this spacecraft position at pixel time, the location of the FOV on the Earth surface is found as the intersection of the antenna beam with the Earth surface following the geolocation procedure described in Hollinger et al. (1989) and Wentz (1991). While comparing the new and the original geolocation, it was found that both usually agree within $\pm 2 \mathrm{~km}$. The deviations can be attributed to simplifications in the operational processor. The original raw data records (RDRs) also show a gradual degradation within a few days followed by sudden jumps when the ephemeris data set was updated in the operational processing.

While the SSM/I had only surface channels, the geolocation procedure for SSMIS must account for three different groups of feedhorns with different reference heights of the geodetic latitude and longitude. The reference heights are selected similar to the operational algorithm:

- imaging channels (SSM/I like, 19-91 GHz) to Earth's surface,

- lower air sounding channels $(50-59,150-183 \mathrm{GHz})$ to $11 \mathrm{~km}$ height,

- upper air sounding channels $(60-63 \mathrm{GHz})$ to $60 \mathrm{~km}$ height.

New spacecraft attitude corrections for pitch and roll for the SSM/I platforms are found using a method similar to the approach of Berg et al. (2013), where the roll angle is estimated from the slope of the mean $T_{\mathrm{B}}$ values along the scan line. Changes in spacecraft roll and pitch have a distinct effect on the vertically polarized $T_{\mathrm{B}}$ values: A change in pitch modifies the curvature along the scan, and a change in roll results in a gradient along the scan. The method from Berg et al. (2013) has been modified to fit both angle offsets, roll and pitch, from the $T_{\mathrm{B}}$ variation along the scan. Instead of using only vertically polarized channels for the minimization procedure as in the original technique (Berg et al., 2013), polarization differences are employed as the horizontally polarized channels are much less affected. This minimizes the influence of cold intrusions at the scan edge. All FOV positions, weighted with the position-dependent variability, can be used to find mean values for pitch and roll offsets. After applying the correction, the $T_{\mathrm{B}}$ variation along the scan appears very similar for all instruments. Corrections for yaw and elevation offsets for the SSM/I instruments are found empirically from a coastline analysis using the highresolution horizontal $85 \mathrm{GHz}$ channel. The implemented attitude corrections for the SSMIS platforms are taken from the operational configuration (Kummerow et al., 2013) without modifications. 
Another geolocation problem that was identified by CM SAF in the raw data records of the SSM/I and SSMIS instruments is an inconsistent handling of leap seconds. During the time period from 1987 to 201513 leap seconds are officially inserted to account for variations in the Earth's rotation in order to keep the Coordinated Universal Time close to the mean solar time (Bulletin C, https://www.iers.org/IERS/EN/ Publications/Bulletins/bulletins.html, last access: 20 January 2020, IERS, 2020). It can take up to $7 \mathrm{~d}$ before a leap second is introduced to the data record. The time difference itself is not problematic if the observation time and the ephemeris time in the TLE sets are updated synchronously. However, a geolocation error of about $7 \mathrm{~km}$ was found in the original geolocation data if this is not the case. This has been corrected in the CM SAF FCDR, and the observation times are changed in a way that all leap seconds are introduced at the correct position.

\subsubsection{Geolocation for SMMR}

The SMMR was observing the Earth surface with a reflector that scanned by oscillating about the vertical axis between azimuth angles of $\pm 25^{\circ}$. Separate radiometers were used for vertical and horizontal polarization only at $37 \mathrm{GHz}$. The channels at other frequencies used a single radiometer time-shared between vertical and horizontal polarization. On the first half of each scan cycle (from right to left), these radiometers measured at vertical polarization, while on the second half of the scan cycle (from left to right) horizontal polarization was measured. The $37 \mathrm{GHz}$ vertical and horizontal polarization were measured on both halves of the scan cycle. This original scan pattern and FOV geolocation are not provided in the level 1B data files. Instead all observations were interpolated to the corresponding $37 \mathrm{GHz}$ positions with 47 scan positions for each half-scan. This FOV geolocation remains unchanged in the FCDR data processing.

The actual spacecraft position is used to fit the SGP4 orbit model and to estimate daily TLE sets. The TLE sets from the full data record are completed with TLE sets from http://www.spacetrack.org/ (last access: 19 August 2019), filtered and smoothed using a moving averaging window of $\pm 7 \mathrm{~d}$ to compile daily element sets for the entire data record period similar to the SSMI(S) procedures. In the following processing step, the new TLE sets are now used to predict the spacecraft position of each scan line. If the predicted and the archived positions are offset by more than $6 \mathrm{~km}$, the scan is marked in the scan quality flag with a geolocation error. Fractional revolution number and local azimuth angles (not available in the level 1B files) are computed using the predicted spacecraft position. Also, scan angles, reflected sunfootprint angles, and EIA are only available at 30 fixed positions per scan line in the level 1B data record. These variables are interpolated to each of the $94 \mathrm{FOV}$ full scan positions.
The SMMR data record contains erroneous data, which have to be flagged in a first processing step during the geolocation. The spacecraft attitude information (roll, pitch, yaw angles) is scanned for zero-filled values and outliers. These angles were used without quality control in the level $1 \mathrm{~B}$ processing to compute the EIAs. Errors in the EIA are mostly caused by anomalies in the attitude angles. Therefore the zero-filled values and outliers in the attitudes angles and the EIA are replaced with interpolated values. However, no extrapolation is applied and incorrect values at the beginning or end of the data files are set to undefined. The original quality flag status is unset accordingly in this case as the original flagging is no longer necessary due to the interpolation.

\subsection{Antenna pattern matching for high-frequency channels}

For the application of geophysical retrieval algorithms, it can be desirable that about the same area of the Earth surface is seen by all channels. Due to different frequencydependent FOV sizes and sampling patterns, the covered area can be significantly different. Also, the FOV positions can be shifted along- and across-track because the SSMIS feedhorns are not exactly co-aligned. For convenience in the application of geophysical retrievals, the high-resolution channels at $85 \mathrm{GHz}$ (SSM/I) and at $91 \mathrm{GHz}$ (SSMIS) are averaged down to the resolution and position of the corresponding $37 \mathrm{GHz}$ FOV. These channels are made available in both the original high resolution and sampling as well as in the lower-resolution and sampling of the $37 \mathrm{GHz}$ channel in the FCDR data files. FOV differences between the lower resolution channels at 19,22 , and $37 \mathrm{GHz}$ are not accounted for.

\subsubsection{SSM/I}

An SSM/I FOV at $85 \mathrm{GHz}$ covers about $20 \%$ of the area sampled at $37 \mathrm{GHz}$. The sampling distance for the $85 \mathrm{GHz}$ channel is $12.5 \mathrm{~km}$ and for the $37 \mathrm{GHz}$ channel $25 \mathrm{~km}$. The high-frequency channels are thus sampled twice as often in the along- and across-track directions compared to all other SSM/I channels. In order to get a comparable coverage with the $85 \mathrm{GHz}$ channels, nine neighbouring pixels of the highresolution scan lines are averaged to match the size of the corresponding $37 \mathrm{GHz}$ pixel. The high-resolution $85 \mathrm{GHz} T_{\mathrm{B}}$ values are averaged with a Gaussian weighted distance $w$ from the centre FOV resembling the $37 \mathrm{GHz}$ main antenna pattern:

$$
\begin{aligned}
T_{\mathrm{B}, \mathrm{lo}}(s, c) & =\sum_{i=-1}^{1} \sum_{j=-1}^{1} w(c, i, j) \cdot T_{\mathrm{B}}\left(h_{s}+i, h_{c}+j\right) \\
& / \sum_{i=-1}^{1} \sum_{j=-1}^{1} w(c, i, j),
\end{aligned}
$$

where $h_{s}=s \cdot 2$ and $h_{c}=c \cdot 2$ are the high-resolution alongtrack and across-track indices respectively and $s$ and $c$ are the 
corresponding low-resolution indices. The Gaussian weights for the surrounding 8 pixels depend on the relative position of the centre pixel along the scan line $c$ due to the distortion of the conical scanning system at the scan edges. An array of precalculated averaging weights is used in the data processing, providing a fixed set of nine specific weighting coefficients for each of the 64 FOVs in the lower SSM/I resolution.

\subsubsection{SSMIS}

Due to the higher frequency, a single FOV at $91 \mathrm{GHz}$ covers only about $17 \%$ of the area sampled at $37 \mathrm{GHz}$. The lower-resolution channels are averaged on board the spacecraft, combining two neighbouring observations. Hence, the $91 \mathrm{GHz}$ channels are sampled twice as often in the alongscan direction compared to the $37 \mathrm{GHz}$ channels, and the actual centre positions are no longer co-registered with the non-averaged high-resolution FOV positions. In order to get a comparable coverage with the $91 \mathrm{GHz}$ channels, six neighbouring beam positions (two along scan, three along track) are averaged to match the resolution of the corresponding $37 \mathrm{GHz}$ footprint. The averaging is performed similar to Eq. (1), by weighting the individual brightness temperatures with their distance $w$ from the centre FOV.

\subsection{Land mask and sea ice detection}

Each FOV is characterized with a surface type classification flag using Climate and Forecast (CF) metadata convention for flag variables. Possible surface types are water, land, coast, sea ice, and sea ice margin. The centre latitude and longitude of the FOV are used to assign the surface type.

The FCDR specific land-sea mask is derived from the Global Land One-km Base Elevation data base (GLOBE Task Team, 1999). This data set is further adjusted to the footprint resolutions by first removing small islands and land masses with a diameter of less than $5 \mathrm{~km}$ for the lowresolution channels and $2 \mathrm{~km}$ for high-resolution channels, treating these areas as open water. This provides the basic land-sea classification. In a second step the coastal areas are defined by expanding the remaining land areas $50 \mathrm{~km}$ (low resolution) or $15 \mathrm{~km}$ (high resolution) into the sea.

To account for the varying sea ice margins, a daily sea ice mask is compiled from the observed $T_{\mathrm{B}}$ values. These maps are created in two steps. First the total sea-ice-covered fraction within a single FOV is computed using the NASA Team sea ice algorithm of Swift et al. (1985). The resulting sea ice observations from all available instruments are then gridded to common daily mean fields on a regular $0.5^{\circ} \times 0.5^{\circ}$ grid. In order to distinguish between short-lived strong rain events and persisting sea ice, which are characterized by similar temperature signatures, only grid boxes with an average sea ice fraction above $15 \%$ for at least 5 consecutive days are flagged as ice covered. This filter removes all events lasting less than $5 \mathrm{~d}$ from the first-guess ice mask. Daily sea ice maps are then derived from this screened data set by re-expanding the reliably identified sea ice areas in time and space while filling remaining data gaps by spatial and temporal interpolation. Finally, the resulting sea ice margin is extended $50 \mathrm{~km}$ into the ocean, which leads to the sea ice and sea ice margin surface types.

\subsection{Radiometric calibration}

The basic assumption for a microwave radiometer calibration is a linear relation of the radiometer output voltage (measured in counts) to the radiometric input, neglecting non-linear effects in the detector. Two reference targets with known temperatures and corresponding radiometer measurements at these temperatures are sufficient for an absolute linear twopoint calibration. The actual implementation of the radiometer differs between the SMMR and SSMI(S) instruments due to the different design.

\subsubsection{SSM/l}

The SSM/I calibration equation is defined as follows (Hollinger et al., 1987):

$T_{\mathrm{A}}=S \cdot C^{\mathrm{e}}+O$,

where $T_{\mathrm{A}}$ is the antenna temperature, $C^{\mathrm{e}}$ denotes the measured counts when the radiometer is switched to the antenna (Earth view), $S$ is the calibration slope, and $O$ is the offset. The warm calibration target of the SSM/I is an internal black-body radiator with three embedded thermistors, constantly measuring its temperature. The cold reference target is a mirror pointing to the cold space, with an assumed constant radiometric background temperature at $2.7 \mathrm{~K}$. The radiometer views the internal warm and cold calibration targets once during a scan rotation. For all seven instrument channels five radiometric readings are taken for both calibration targets during an A scan, and five additional measurements are taken for the two $85 \mathrm{GHz}$ channels during a B scan. The three embedded thermistor readings are available once per scan pair. The calibration slopes $S$ in kelvin per count and offsets $O$ in kelvin for each instrument channel are calculated following Hollinger et al. (1987) as follows:

$$
\begin{aligned}
S & =\frac{\left\langle T^{\mathrm{h}}\right\rangle-T^{\mathrm{c}}}{\left\langle\overline{C^{\mathrm{h}}}\right\rangle-\left\langle\overline{C^{\mathrm{c}}}\right.}, \\
O & =\frac{T^{\mathrm{c}} \cdot\left\langle\overline{C^{\mathrm{h}}}\right\rangle-\left\langle T^{\mathrm{h}}\right\rangle \cdot\left\langle\overline{C^{\mathrm{c}}}\right\rangle}{\left\langle\overline{C^{\mathrm{h}}}\right\rangle-\left\langle\overline{C^{\mathrm{c}}}\right\rangle},
\end{aligned}
$$

where $\left\langle\overline{C^{\mathrm{h}}}\right\rangle$ denotes smoothed mean warm counts, $\left\langle\overline{C^{\mathrm{c}}}\right\rangle$ denotes smoothed mean cold counts, $\left\langle\overline{T^{\mathrm{h}}}\right\rangle$ denotes smoothed 
mean warm target temperature, and $T^{\mathrm{c}}$ is the cold target temperature. The smoothed scan line mean values for the cold counts $C^{\mathrm{c}}$, the warm counts $C^{\mathrm{h}}$, and the warm target temperature $T^{\mathrm{h}}$ are derived by first averaging all available measurements for each scan pair that passes the quality control:

$$
\begin{aligned}
& \overline{C^{\mathrm{h}}}=\frac{1}{n} \cdot \sum_{s=1}^{n} C_{s}^{\mathrm{h}}, \\
& \overline{C^{\mathrm{c}}}=\frac{1}{n} \cdot \sum_{s=1}^{n} C_{s}^{\mathrm{c}}, \\
& \overline{T^{\mathrm{h}}}=\frac{1}{3} \cdot \sum_{s=1}^{3} T_{s}^{\mathrm{h}},
\end{aligned}
$$

where $C_{s}^{\mathrm{c}}, C_{s}^{\mathrm{h}}$, and $T_{s}^{\mathrm{h}}$ are the individual samples of the warm target, the cold target, and the warm load target thermistors respectively. In order to further reduce the noise in the calibration input values, an additional Gaussian smoothing of these scan line mean values, denoted with the \langle\rangle operator is applied:

$$
\begin{aligned}
\left\langle\overline{C^{\mathrm{h}}}\right\rangle & =\sum_{i=s-g}^{s+g} w_{i} \cdot \overline{C^{\mathrm{c}}} i / \sum_{i=-g}^{g} w_{i}, \\
\left\langle\overline{C^{\mathrm{c}}}\right\rangle & =\sum_{i=s-g}^{s+g} w_{i} \cdot \overline{C^{\mathrm{c}}} i / \sum_{i=-g}^{g} w_{i}, \\
\left\langle\overline{T^{\mathrm{h}}}\right\rangle & =\sum_{i=s-g}^{s+g} w_{i} \cdot \overline{T^{\mathrm{h}}} i / \sum_{i=-g}^{g} w_{i},
\end{aligned}
$$

where $w_{i}$ denotes the Gaussian across-scan weighting coefficients. The moving averaging kernel spans the $g$ preceding and $g$ following mean scan line values. The kernel half-size $g$ is set to 5 for all SSM/I sensors. This smoothing process introduces an artificial error correlation in the smoothed calibration coefficients. However, as the variance of the calculated $T_{\mathrm{B}}$ values is dominated by the variance in the independently measured Earth's counts $C^{\mathrm{e}}$, the impact of the error correlation in the smoothed calibration coefficients on the final brightness temperatures can be neglected.

The temperature of the warm target $T^{\mathrm{hl}}$ needs to be corrected using the temperature of the forward radiator plate $T^{\mathrm{pl}}$ to account for radiative coupling between the warm load and the top plate of the rotating drum assembly which faces the warm load when not being viewed:

$T^{\mathrm{h}}=\varepsilon \cdot T^{\mathrm{hl}}+(1-\varepsilon) \cdot T^{\mathrm{pl}}$

The value for $\varepsilon$ is empirically determined from thermalvacuum calibration (Hollinger et al., 1987). The original value of $\varepsilon=0.99$ has been changed to sensor-specific values determined during the inter-sensor calibration procedure (see Sect. 5) for this FCDR. Calculated slopes and offsets are archived in the FCDR data files for traceability. The antenna temperatures are then calculated from the Earth counts $C^{\mathrm{e}}$ for each FOV using Eq. (2). Keeping the calibration coefficients in the data record allows us to revert the original Earth counts from the temperatures.

\subsubsection{SSMIS}

The calibration of SSMIS follows the SSM/I calibration with Eqs. (2) and (3). The cold and warm calibration view counts and the warm target temperature are already averaged by the SSMIS on-board software and are down-linked as scan line mean values. The across-scan averaging kernel half-width $g$ in Eq. (5) is set to eight scan lines for channels 1-7, four scan lines for channels 8-18, and 32 scan lines for channels 19-24. This setup also corresponds to the latest operational ground processing software configuration (Kummerow et al., 2013).

The configuration of the on-board averaging software has changed over time for SSMIS $\mathrm{F}_{16}$ and SSMIS $_{\mathrm{F} 17}$. The original setup was to average the radiometric readings of the cold and warm target of the current scan and the seven preceding scan lines and is thus asymmetric. Because this configuration resulted in a striping pattern, the on-board acrossscan averaging was eventually switched off after revolution number 29808 for SSMIS $_{\mathrm{F} 16}$ and revolution number 1062 for $\mathrm{SSMIS}_{\mathrm{F} 17}$. In order to get a symmetric average of the calibration counts for the time period when only the asymmetric mean values are available, the averaging window for channels $8-18$ must be expanded to a minimum of 14 scans. This results from averaging the two eight-scan mean values at scan $n$ and at scan $n+7$. This larger averaging kernel size reduces the noise in the affected SSMIS $_{\mathrm{F} 16}$ and SSMIS $_{\mathrm{F} 17}$ channels during the aforementioned time period.

\subsubsection{SMMR}

The calibration method applied in the Pathfinder level 1B data record for SMMR was taken from the SMMR CELL data record (Fu et al., 1988):

$T_{\mathrm{A}}=I+S \cdot\left(\frac{C^{\mathrm{e}}-C^{\mathrm{h}}}{C^{\mathrm{c}}-C^{\mathrm{h}}}\right)$,

where $T_{\mathrm{A}}$ is the antenna temperature, and $C^{\mathrm{e}}, C^{\mathrm{h}}$, and $C^{\mathrm{c}}$ are the measured counts when the radiometer is switched to the antenna (Earth view), the warm load calibration, and cold load calibration respectively. The calibration coefficients $S$ and $I$ are defined as functions of the instrument's component temperatures:

$$
\begin{aligned}
& I=a_{1}+a_{2} T_{\mathrm{sw}}-\Delta_{\mathrm{fh}}, \\
& S=a_{3}\left(T_{\mathrm{sw}}-2.7\right)+\Delta_{\mathrm{ch}} .
\end{aligned}
$$

The $\Delta_{\mathrm{fh}}$ term is a correction for temperature gradients from the multi-frequency feedhorn along the feedhorn waveguide 
to the receiver and is given by

$\Delta_{\mathrm{fh}}=\left(T_{\mathrm{sw}}-T_{\mathrm{fh}}\right)-\left(\frac{T_{\mathrm{fw}}-T_{\mathrm{fh}}}{\alpha_{1}}\right)+\left(\frac{T_{\mathrm{sw}}-T_{\mathrm{fw}}}{\alpha_{1} \beta_{1}}\right)$,

with $T_{\mathrm{sw}}, T_{\mathrm{fh}}$, and $T_{\mathrm{fw}}$ as the Dicke switch, feedhorn, and feedhorn waveguide temperatures respectively.

The $\Delta_{\mathrm{ch}}$ term is a correction for temperature gradients from the cold-sky calibration horn along the calibration horn waveguide to the receiver and is given by

$\Delta_{\mathrm{ch}}=\left(T_{\mathrm{sw}}-T_{\mathrm{ch}}\right)-\left(\frac{T_{\mathrm{cw}}-T_{\mathrm{ch}}}{\alpha_{2}}\right)+\left(\frac{T_{\mathrm{sw}}-T_{\mathrm{cw}}}{\alpha_{2} \beta_{2}}\right)$,

with $T_{\mathrm{ch}}$ and $T_{\mathrm{cw}}$ as the calibration horn and calibration horn waveguide temperatures. The calibration coefficients $a_{1,2,3}$, $\alpha_{1,2}$, and $\beta_{1,2}$ are given in Njoku et al. (1998).

The warm calibration target of the SMMR is the internal ambient temperature at $\approx 300 \mathrm{~K}$. The cold reference target is the cold space, viewed with three calibration horns (depending on frequency). The cold sky is assumed to be at a constant temperature of $2.7 \mathrm{~K}$, i.e. cosmic background temperature. The radiometer views the internal warm and cold calibration targets once during a full scan cycle. The instrument temperatures are sampled every eight scans using platinum resistance thermistors and are embedded in the data record.

In order to reduce the noise, calibration warm and cold counts are smoothed in the level $1 \mathrm{~B}$ data processing using a running one-sided average:

$\left\langle C^{\mathrm{h}, \mathrm{c}}(s)\right\rangle=w \cdot C^{\mathrm{h}, \mathrm{c}}(s)+(1-w) \cdot\left\langle C^{\mathrm{h}, \mathrm{c}}(s-1)\right\rangle$,

with $s$ as the scan line number, $w$ as the weight of the new data value, and $\left\langle C^{\mathrm{h}, \mathrm{c}}\right\rangle$ as the across-scan smoothed cold or warm count. A weight of $w=0.1$ was chosen as a compromise between noise reduction and overdamping.

Also, the instrument temperatures are averaged using the same method. As these temperatures are only available every eight scans, a weight of $w=0.6$ was used.

The calibration coefficients $S$ and $I$ are not available in the Pathfinder level 1B data records. In order to complete the data record and to estimate the instrument noise level, these coefficients are recalculated using Eqs. (8)-(10) and then archived in the SMMR FCDR data files for reference.

\subsection{Corrections applied to raw data records}

A number of technical issues affecting the quality of the measurements have been identified for all radiometers. They have to be corrected as one major part of the FCDR generation before an inter-calibration can be derived.

\subsubsection{SSM/I along-scan correction}

One of the first detected SSM/I calibration problems was a non-uniformity of the measured antenna temperatures along a scan line (Wentz, 1991; Colton and Poe, 1999). A rapid falloff at the end of the Earth scan of nearly 1.5 to $2 \mathrm{~K}$ was found in all SSM/I channels. This systematic problem is caused by an intrusion of cosmic background energy by the glare Suppression System-B (Colton and Poe, 1999) into the antenna feedhorn reducing the observed scene temperature and thus causing a scan-position- and scene-temperature-dependent offset.

This intrusion effect can be treated as an antenna beam position energy loss. It can be corrected by multiplying the antenna temperature $T_{\mathrm{A}}$ with a scan-position-dependent correction factor. This factor is derived in a two-step procedure. First, antenna temperatures for each sensor and channel with surface type classified as sea between $50^{\circ} \mathrm{S}$ and $50^{\circ} \mathrm{N}$, thereby excluding areas with seasonally varying sea ice, are normalized to a constant Earth incidence angle (EIA). Then these temperatures are averaged into FOV-position bins for at least 1 year. The correction factor at each FOV position is computed as the ratio of the position averaged value to the average along the unaffected FOVs positions at the scan line centre (FOV positions 50-90). The derived along-scan correction factors are very similar for all SSM/I sensors. As an example, the along-scan correction for $\mathrm{SSM} / \mathrm{I}_{\mathrm{F} 13}$ is shown in Fig. 2. The see-saw pattern in the $85 \mathrm{GHz}$ channels results from two sets of integrators being used, one for the odd and one for the even numbered positions with small differences remaining in the level 0 data.

\subsubsection{SSM/I cold-space reflector intrusions}

The SSM/I calibration procedure assumes that the cold reference target is at the background cosmic temperature of $2.7 \mathrm{~K}$. However, due to the open instrument design, it is possible, depending on the orbit parameters, that the moon is visible in the cold view. This leads to a short-term increase in the measured cold target radiation counts. The amplitude and length of this increase depend on the beam width of the channel as the fraction of the moon in the FOV compared to the cosmic background is larger for smaller beam widths. These events usually happen twice per month, depending on the orbit configuration. An example is shown in Fig. 3. This image is a two-dimensional representation of cold count anomalies as a function of the northward Equator crossing time on the $x$ axis and the fractional orbit position on the $y$ axis. One image column is exactly one orbit with the start of the orbit at the time given on the abscissa, and one image row is always at the same orbit angle position. The image depicts two intrusion events, the first one over the North Pole on 14 November and the second one on 19 November shortly before crossing the South Pole.

As these events only affect the cold reference target, the impact on the calibrated antenna temperatures is scene dependent and larger for lower scene temperatures. The best way to correct this effect is to apply a band-pass filter to remove the additional signal induced from the moonlight. Un- 


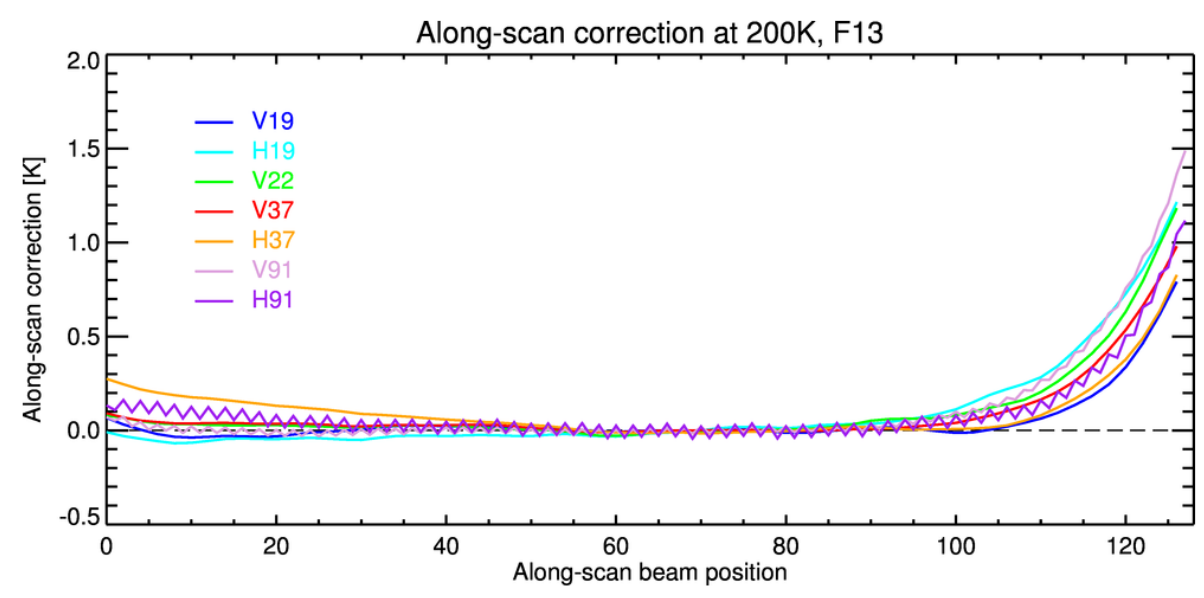

Figure 2. Along-scan correction for SSM/I $\mathrm{I}_{\mathrm{F} 13}$. The image shows the along-scan correction applied to a scene temperature of $200 \mathrm{~K}$. Colours are as follows: blue - v19; cyan - h19; green - v22; red - v37; orange - h37; plum - v85; violet - h85.
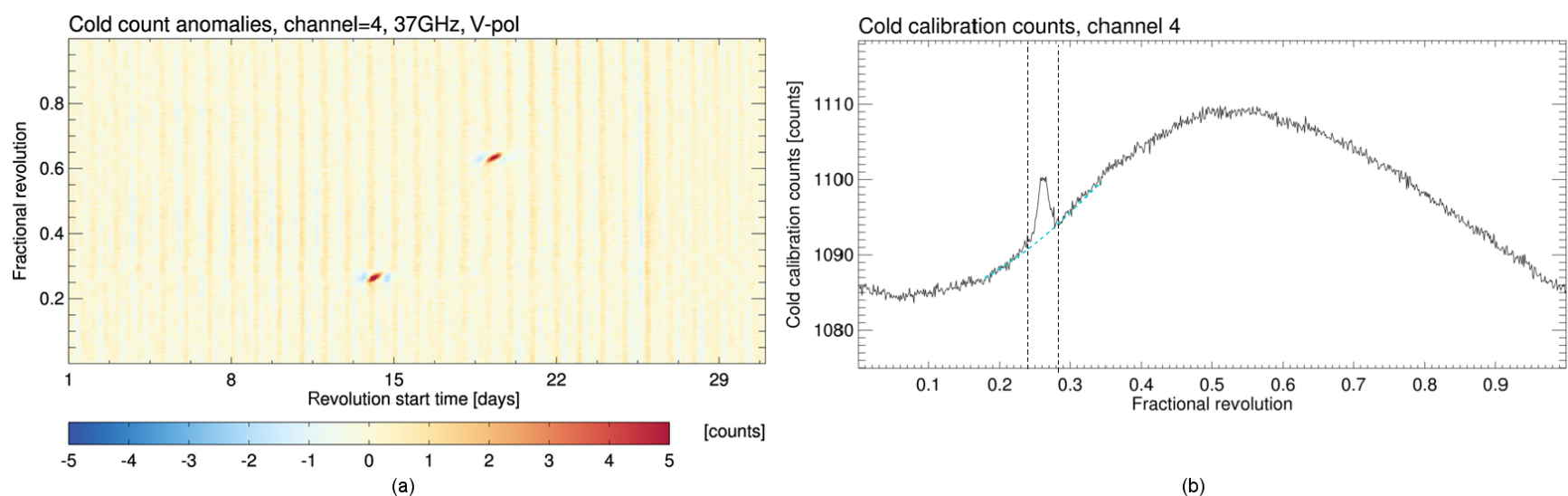

(b)

Figure 3. Cold count anomaly (a) from the SSM/I 13 channel at $37 \mathrm{GHz}$, v-pol for November 2005. The $y$ axis represents the fractional orbit angle with an ascending Equator crossing at 0 and descending Equator crossing at 0.5. The $x$ axis represents the time of the orbit start at the ascending Equator crossing. A cross section of the absolute cold counts through the maximum of the first intrusion event is shown in (b). Positions with identified cold count anomalies are between the vertical lines. The locally fitted reconstruction spline is plotted in cyan.

der normal conditions the variation in the cold counts is periodic and changes very little from orbit to orbit. This allows us to easily detect the anomalies as shown in Fig. 3.

A procedure has been developed which automatically detects the moonlight intrusion events using a Laplace filter as an edge detection algorithm. The Laplace operator computes the second derivatives of an image, measuring the rate at which the first derivatives change. This determines if a change in adjacent pixel values is from an edge or continuous progression. In order to use standard image filtering techniques to detect the intrusion events, yearly monitoring files are compiled for each sensor and channel. The calibration data are sampled at fixed orbit angle positions. The discrete cold count Laplace operator $\Delta^{\mathrm{c}}$ is then defined as with $i$ and $j$ as the along-orbit and across-orbit image indices respectively, $k$ as the kernel size, and $\widehat{C}^{\mathrm{c}}$ as the sampled cold counts. This image is then smoothed and periods with moonlight intrusion are detected where the derived Laplacian is significantly larger then 1 standard deviation of the background field. These periods are regarded as missing data and reconstructed along the orbit using a spline interpolation procedure from the neighbouring smoothed valid values (see Fig. 3, cyan line). The difference between the original and the reconstructed values is smoothed and removed from the original values. This procedure works similarly to a local bandpass filter but can also deal with undefined data. Corrected scan lines are also flagged during the final data processing using a processing control flag.

$\Delta_{i, j}^{\mathrm{c}}=2 \cdot \widehat{C}_{i, j}^{\mathrm{c}}-\widehat{C}_{i-k / 2}^{\mathrm{c}}-\widehat{C}_{i+k / 2}^{\mathrm{c}}$, 


\subsubsection{SSM/I warm load intrusions}

Another problem in the SSM/I calibration data that has been identified is sunlight intrusion into the warm reference blackbody target. This occurs during certain orbit constellations when the satellite platform is leaving and entering the Earth shadow.

The issue is caused by an illumination of the black-body tines due to either direct or reflected sunlight. The sunlight causes a fast instant response in the calibration measurements of the SSM/I as the radiation heats the tines of the black body while the black-body core remains unaffected or is just slowly heating up. As the warm load temperature sensors are embedded into the core of the black body, they do not measure the temperature of the calibration target at the tines but the core temperature. With the delayed response of the temperature sensors to the increased radiation, the net effect is a sharp artificial discontinuity (decrease) in the calibration gain. Figure 4 shows an example for the detection of intrusion events. The intrusions occur around the orbit positions 0.23 and 0.81 . When the satellite leaves the Earth's shadow, the whole instrument warms up, and the warm load temperature also increases. When the intrusion ceases, the black-body core and surface temperature are balanced again (around position 0.32). However, if the satellite enters the Earth's shadow, the intrusion effect is more pronounced. As long as the sunlight falls on the warm load, a negative anomaly can be observed in the calibration slopes, because the core temperature of the black body remains nearly constant. When the sunlight intrusion vanishes (around position 0.9) a sharp increase is visible in the slope when measured warm load target temperature and the observed radiation are balanced again.

This sunlight intrusion effect depends on the channel frequency and is strongest at the lower frequencies. The procedure to correct for this intrusion is similar to the correction for the moonlight intrusions, but in the sunlight case the warm target counts are affected. All significant sunlight intrusion events are detected using the edge detection algorithm applied to the slope anomalies. The discrete Laplace operator $\Delta^{s}$ is applied to the sampled calibration slopes $\widehat{S}$ :

$\Delta_{i, j}^{s}=2 \cdot \widehat{S}_{i, j}-\widehat{S}_{i-k / 2}-\widehat{S}_{i+k / 2}$.

The intrusion periods are filtered and reconstructed from a spline interpolation using the neighbouring smoothed valid slope values (see Fig. 4, cyan line). The difference between the original and the reconstructed slope values is smoothed to obtain the intrusion-induced anomaly and then used to correct the computed slopes. Finally the corrected slopes and the warm load calibration temperature are used to convert the slope anomaly into warm load count offset maps. These corrections are applied during the final data processing to correct the intrusion events. Affected scans are also flagged using a processing control flag.
An example for the edge detection algorithm (applied Laplace operator) is shown in Fig. 4. The positions of the sunlight intrusion events are identified as positive and negative anomaly lines. The geographic positions of the intrusions depict a seasonal cycle caused by the varying relative angle of the satellite to the sun. The pattern also changes from year to year due to the orbit drift and changing Equator crossing times. Therefore the correction maps are compiled for each year separately.

\subsubsection{SSMIS along-scan correction}

Similar to the non-uniformity of the measured antenna temperatures along a scan line for SSM/I, the SSMIS sensors also show a rapid decrease near the end and beginning of the Earth-viewing scene $T_{\mathrm{B}}$ values (Kunkee et al., 2008b). A rapid fall-off at the end of the Earth scan is found for channels 15 and $16(37 \mathrm{GHz})$, due to intrusions into the feedhorn by the warm load cover and for the beginning of the scan line for channels 12-14 (19 and $22 \mathrm{GHz}$ ), due to intrusions by the cold-sky reflector. The strongest effect can be observed for the SSMIS ${ }_{\mathrm{F} 17}$ channels $15-16$, when the last five beam positions are biased by more than $8 \mathrm{~K}$.

This effect is corrected following the approach for SSM/I by multiplying the antenna temperature with a scan-positiondependent correction factor. The along-scan correction for the SSMIS $\mathrm{F} 16_{6}$ instrument is exemplarily shown in Fig. 5.

\subsubsection{SSMIS cold-space reflector intrusions}

Due to the heritage design from the SSM/I, the moonlight intrusions into the cold-space reflector are also inherited by SSMIS. The correction procedure principally follows the one from the SSM/I. However, the SSMIS consists of six individual feedhorns with slightly different alignments. This leads to a small shift in the location of the intrusion, which has to be accounted for, and the correction is carried out for each feedhorn separately.

\subsubsection{SSMIS warm load intrusions}

As with the cold-space reflector intrusions, the sunlight intrusion into the warm calibration black-body target is also inherited by the SSMIS. In fact, the warm load intrusion were

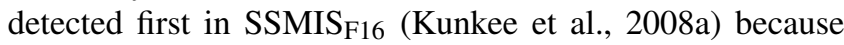
they are stronger and cover a significantly larger part of the orbit. Kunkee et al. (2008a) identified two intrusion regions where the sun directly shines on the warm load tines and two regions where the radiation is reflected from the top of the SSMIS canister onto the warm load target. In order to mitigate the direct intrusions, a fence was installed aboard F17 and F18.

Following the approach developed for SSM/I, the sunlight intrusion events for the SSMIS are detected by applying the Laplace operator Eq. (13) to the calibration slope. An exam- 


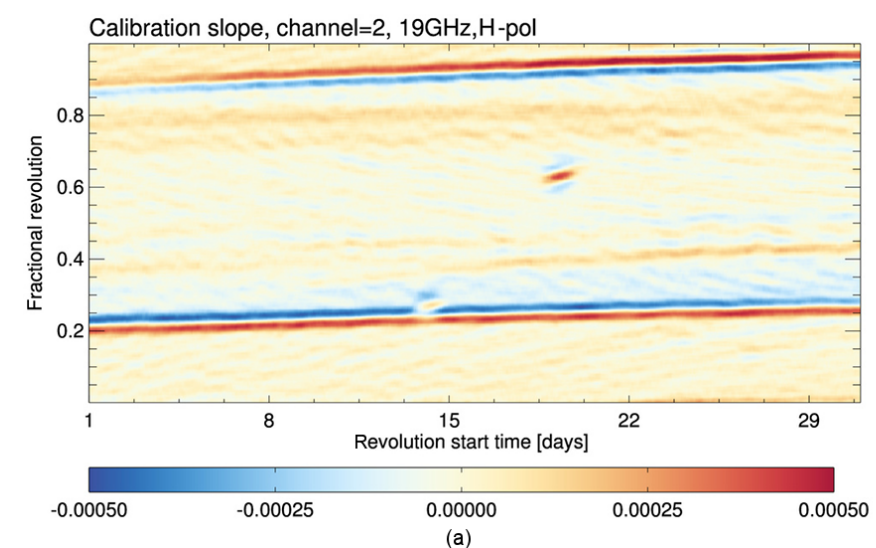

(a)

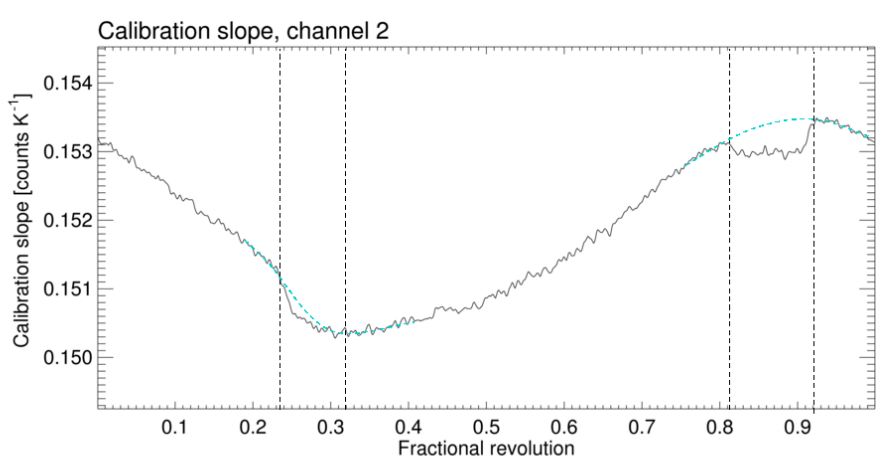

(b)

Figure 4. Edge detection results (Laplace operator) for calculated first-guess slopes from the SSM// $\mathrm{F}_{13}$ channel at $19 \mathrm{GHz}$, h-pol for November 2005 (a) and a cross section of the first-guess slope (b) with two sunlight intrusion events. The satellite leaves the Earth's shadow at approximately position 0.28 and enters the Earth's shadow at position 0.85 . Positions with identified warm target anomalies are between the vertical lines. The locally fitted reconstruction splines are plotted in cyan.

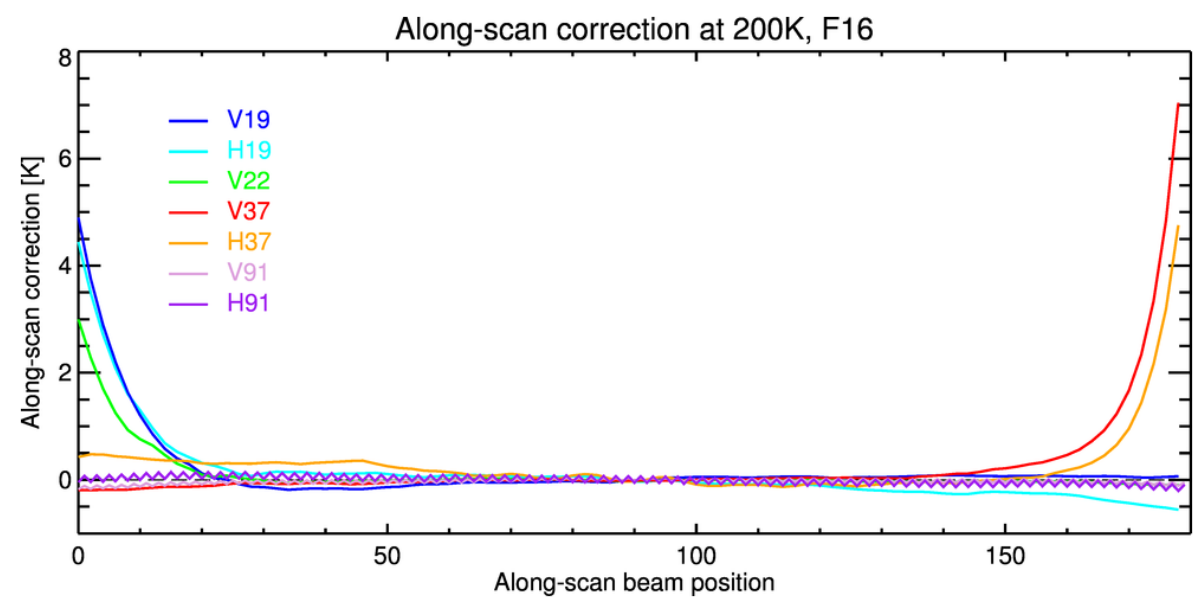

Figure 5. Along-scan correction for SSMIS $\mathrm{F16}$. The image shows the along-scan correction applied to a scene temperature of $200 \mathrm{~K}$. Colours are as follows: blue - v19; cyan - h19; green - v22; red - v37; orange - h37; plum - v91; violet - h91.

ple showing four sunlight intrusion events at orbit positions $0.04,0.15,0.48$, and 0.65 is presented in Fig. 6 for SSMIS $\mathrm{F} 16$. Apart from the regularly repeated short-term moonlight intrusion events, which occur around orbit positions 0.3 and 0.7 , strong regular stripes can be identified in Fig. 6a. The negative anomaly regions in the Laplacian, flanked with positive anomalies, are the regions with sunlight intrusions affecting the calibration gain. These patterns are not static at a geographic position but vary during the year as a function of the solar elevation and azimuth angles relative to the spacecraft platform. Also, spacecraft structure elements, like solar panels, can shade the warm load during some periods of the year. This can be observed in February (March) around orbit position 0.5 , where the anomaly abruptly stops (starts). The additional fence on DMSP F17 and F18 is working well (not shown here). Only during a 3-month period, when the sun is above the installed fence, is an intrusion visible for $\mathrm{SSMIS}_{\mathrm{F} 17}$, and just a small intrusion region for $\mathrm{SSMIS}_{\mathrm{F} 18}$ can be identified throughout the year. The basic correction method again follows that of SSM/I. However, as a consequence of the aforementioned factors, it is not possible to compile one static intrusion map for each channel; instead yearly correction maps are compiled for each channel. In order to minimize the noise, the SSM/I procedure has been adapted to convert the observed count anomalies into an anomaly in the warm target temperature first. These temperature anomalies are then averaged across all channels sharing one feedhorn and eventually converted back to channelspecific count anomalies.

\subsubsection{SSMIS emission from main reflector}

The SSMIS main reflector appears to be emissive (Bell, 2008; Kunkee et al., 2008a), which results in an additional 

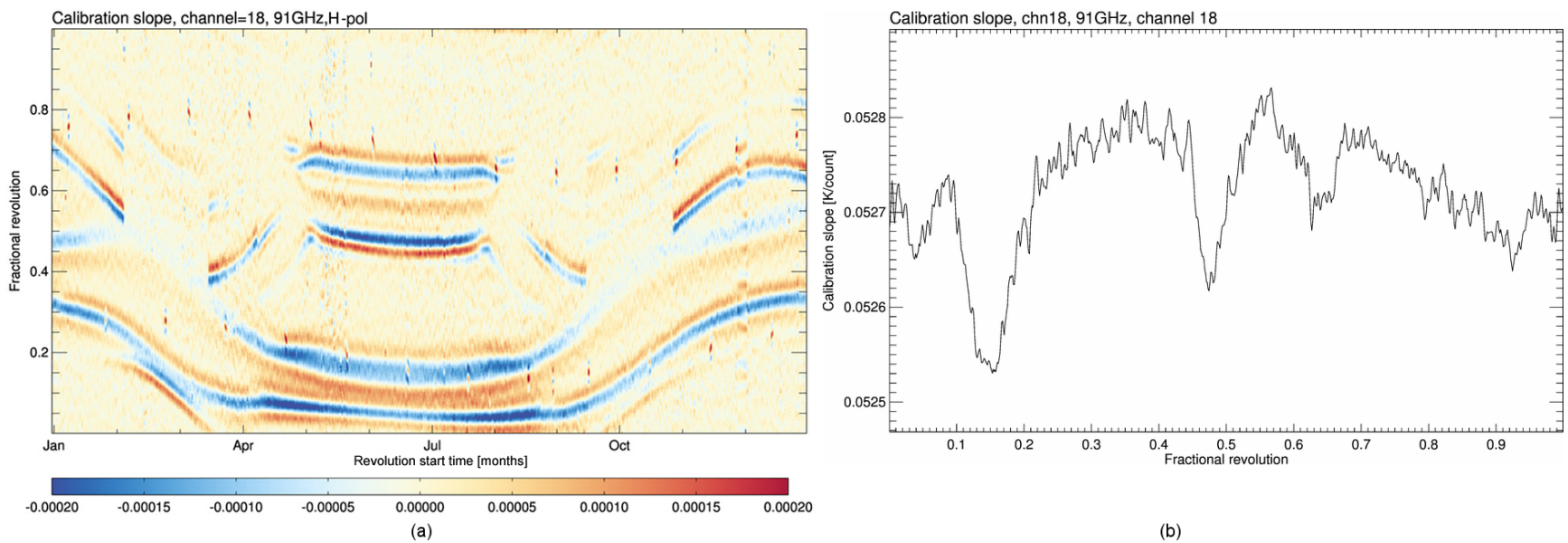

Figure 6. Edge detection results (Laplace operator) for calculated first-guess slopes from the SSMIS $\mathrm{F} 16$ channel at $91 \mathrm{GHz}$, h-pol for 2010 (a) and a cross section of first-guess slope (b) for one orbit in June 2010 (right). Four regions with warm load intrusion are identified around orbit positions $0.04,0.15,0.48$, and 0.65 .

radiation emitting from the reflector at the reflector's temperature and thus modifying the measured scene temperature. The magnitude of this effect depends on the temperature difference between the reflector and the Earth scene. The reflector temperature varies along the orbit due to solar heating when not being covered in the Earth shadow. The strongest signal can be observed when the spacecraft leaves the Earth shadow and the reflector temperature increases by about $80 \mathrm{~K}$ within a few minutes.

If the temperature of the main reflector $T_{\text {refl }}$ and its emissivity $\varepsilon_{\text {refl }}$ are known, the emission effect can be corrected with

$T_{\mathrm{A}}^{\prime}=\frac{T_{\mathrm{A}}-\varepsilon_{\mathrm{refl}} \cdot T_{\mathrm{refl}}}{1-\varepsilon_{\mathrm{refl}}}$

Values for $\varepsilon_{\text {refl }}$ were found by comparing observed minus background residuals from data assimilation experiments by Bell (2008). Emissivity values derived for the Universal Preprocessor (UPP) version 2 are applied in this FCDR unchanged for all non SSM/I-like channels. Emissivity values for the SSM/I-like channels are fitted again during the intercalibration minimization procedure because the given values turned out to be not at the required precision. The values are determined by using SSMIS $_{\mathrm{F} 18}$ as the reference target, because no significant emissivity was found for its reflector.

From SSMIS $\mathrm{F} 17_{7}$ onward, a temperature sensor is placed at the back of the reflector to measure $T_{\text {refl }}$ directly, but for SSMIS $_{\mathrm{F} 16}$ the closest available sensor is placed on the rim of the main reflector, close to the support arm. The temperature of the reflector can be constructed from the arm temperature $T_{\text {arm }}$ using a lagged correction following Bell (2008). The reconstructed reflector temperature is computed for F16 and saved in the FCDR data files. The reflector temperature for all sensors is linearly interpolated to the observation time as the housekeeping data records are not available for each scan line and the antenna temperatures are corrected with Eq. (14).

\subsubsection{SMMR Pathfinder level 1B corrections}

In the level 1B processing, corrections for absolute calibration, long-term drifts, short-term drifts, and polarization mixing are applied. The level $1 \mathrm{~B}$ corrections applied to the antenna temperatures are explained in detail in Njoku et al. (1998) and summarized in the following two subsections.

\section{(a) SMMR calibration offset adjustments}

A two-point linear absolute calibration adjustment to the SMMR data record was estimated by Njoku et al. (1998) using ocean areas acting as the cold calibration target and the internal ambient instrument warm load as the warm calibration target. The coefficients were derived over calm, cloudfree oceanic areas, characterized by low emissivity values. Modelled $T_{\mathrm{B}}$ values were compared to the observed SMMR $T_{\mathrm{B}}$ values to derive a linear calibration adjustment to the antenna temperatures:

$T_{\mathrm{A}_{c}}^{\prime}=a+b \cdot T_{\mathrm{A}}^{\prime}$,

where $T_{\mathrm{A}}^{\prime}$ is the normalized SMMR antenna temperature (after spillover correction), $T_{\mathrm{A}_{c}}^{\prime}$ is the corrected antenna temperature value, and $a, b$ are the linear calibration adjustment coefficients. The calibration constraints can be written as

$T_{\mathrm{B}}^{\mathrm{m}}=T_{\mathrm{B}_{c}}^{\mathrm{o}}=a+b \cdot T_{\mathrm{B}}^{\mathrm{o}}$,

$I^{\prime}=a+b \cdot I^{\prime}$,

where $T_{\mathrm{B}}^{\mathrm{o}}$ is the mean observed SMMR brightness temperature for the cold calibration target and $T_{\mathrm{B}}^{\mathrm{m}}$ is the correspond- 
ing modelled brightness temperature. Solving these equations for the coefficients $a$ and $b$ yields

$a=I^{\prime} \cdot \frac{T_{\mathrm{B}}^{\mathrm{m}}-T_{\mathrm{B}}^{\mathrm{o}}}{I^{\prime}-T_{\mathrm{B}}^{\mathrm{o}}}$,

$b=\frac{I^{\prime}-T_{\mathrm{B}}^{\mathrm{m}}}{I^{\prime}-T_{\mathrm{B}}^{\mathrm{o}}}$.

The calibration correction coefficients $a$ and $b$ depend on the warm load equivalent brightness $I^{\prime}$ and are updated online for each new set of calibration data. The values for $T_{\mathrm{B}}^{\mathrm{m}}$ and $T_{\mathrm{B}}^{\mathrm{o}}$ are given in Njoku et al. (1998).

\section{(b) SMMR correction for short- and long-term drifts}

Ageing of the radiometer components can lead to a slow but steady degradation in the radiometer performance. Njoku et al. (1998) studied the long-term evolution of the mean $T_{\mathrm{B}}$ values in all SMMR channels. The largest drift was found for the channel at $21 \mathrm{GHz}$, v-pol with an increase of $36 \mathrm{~K}$ until March 1985 when it was turned off. The observed drifts are corrected in the level 1B data record using a polynomial fit of the globally averaged antenna temperatures, after removing mean seasonal cycle variations. The polarization mixing was not corrected for in order to keep the signals from each radiometer independent at this stage. Otherwise the strong drift in the horizontal polarization at $21 \mathrm{GHz}$ would have been mixed into the vertical polarized channel.

Also, orbit-dependent solar illumination of the instrument can affect the radiometric calibration. Although temperature gradients are accounted for in the SMMR calibration using Eqs. (7) and (8), not all effects might be compensated accurately. Especially when the spacecraft leaves or enters the Earth's shadow, strong temperature gradients can be observed, possibly leading to unmodelled thermal emissions in the waveguides and switches of the instrument (Francis, 1987).

To correct for this type of errors, orbit-dependent corrections were derived following a method described by Francis (1987). Mean brightness temperature differences between ascending and descending orbits were analysed to derive $T_{\mathrm{B}}$ drift corrections as a function of the spacecraft ecliptic angle. The corrections are derived for each year and then linearly interpolated to the observation time.

The corrections for long-term drifts $T_{\text {lt }}$ and short-term drifts $T_{\text {st }}$ were incorporated into the calibration by replacing the calibration correction coefficients $a$ and $b$ in Eq. (17) with drift-corrected coefficients $a_{n}$ and $b_{n}$. However, as the original coefficients $a_{n}$ and $b_{n}$ are not available in the data records and the short-term and long-term correction maps are not available in Njoku et al. (1998), it is not possible to undo the applied corrections and to revert the original antenna temperatures. Therefore, any other additional correction must be carried out on top of the already applied level 1B corrections.

\subsubsection{SMMR along-scan correction}

A general along-scan non-uniformity of the SMMR antenna temperatures is to be expected, due to the design of the radiometer, causing a scan-angle-dependent cross-polarization coupling. Although most of this effect is removed by the applied scan-position-dependent polarization mixing correction (see Sect. A3), significant variations in the final $T_{\mathrm{B}}$ values can remain. To analyse these, the match-up data set with observed and modelled brightness temperatures from the inter-calibration procedure (see Sect. 5.3) has been used here.

Differences between observed and modelled $T_{\mathrm{B}}$ values for each channel with surface type classified as sea between $50^{\circ} \mathrm{S}$ and $50^{\circ} \mathrm{N}$ are averaged into FOV position bins for the complete SMMR time period at $10 \mathrm{~d}$ intervals. Then a factor at each FOV position is computed as the ratio of the position-averaged value to the unaffected FOVs at the scan line centre (azimuth $=0$ ). The time mean results are shown in Fig. 7 for a nominal scene temperature at $200 \mathrm{~K}$. Most pronounced features are the large offsets of about $1 \mathrm{~K}$ for the channel at $21 \mathrm{GHz}, \mathrm{v}$-pol on the left scan side and for the channel at $37 \mathrm{GHz}$, h-pol on the right scan side. The other channels show small offsets on the left side $(0-0.3 \mathrm{~K})$ and small but a little larger offsets at the right side $(0.2-0.5 \mathrm{~K})$. The large differences at $21 \mathrm{GHz}, \mathrm{v}$-pol are not constant over time (not shown here) and are most likely caused by the polarization mixing from the problematic channel at $21 \mathrm{GHz}$, hpol. This channel depicts a strong trend and degradation with time, which can not be removed completely by the long-term drift corrections and polarization mixing decoupling procedure. Also, the observed differences in the $18 \mathrm{GHz}$ channels are not constant over time, but they do show a seasonal variation with smaller offsets in Northern Hemisphere winter and larger offsets in Northern Hemisphere summer months. Only the deviations in the $37 \mathrm{GHz}$ channels are constant over time.

Following these results, only the along-scan biases in the $37 \mathrm{GHz}$ channels are corrected in the CM SAF SMMR FCDR. As the differences in the vertical polarization are much smaller than in the horizontal polarization, the crosspolarization is assumed to be zero and the bias correction reduces to a scan-position-dependent factor similar to the SSMI(S) correction procedure.

\section{Computation of brightness temperatures}

When a space-borne radiometer is observing the Earth surface, the antenna boresight direction can be defined as the unit vector $\widehat{\boldsymbol{s}}_{0}$ from the spacecraft to the Earth surface. The brightness temperature $T_{\mathrm{B}}$ at a polarization $p$ propagating to the antenna from a surface point $\rho$ is then defined as $T_{\mathrm{B}_{p}}(\rho, \widehat{s})$, where $\widehat{\boldsymbol{s}}$ is the unit vector from the antenna to surface at an angle $d \Omega$. Following Stogryn (1978), the corresponding antenna temperature $T_{\mathrm{A}_{p}}\left(\widehat{\boldsymbol{s}_{0}}\right)$ can be expressed as an integral of the normalized co-polarized antenna pattern 


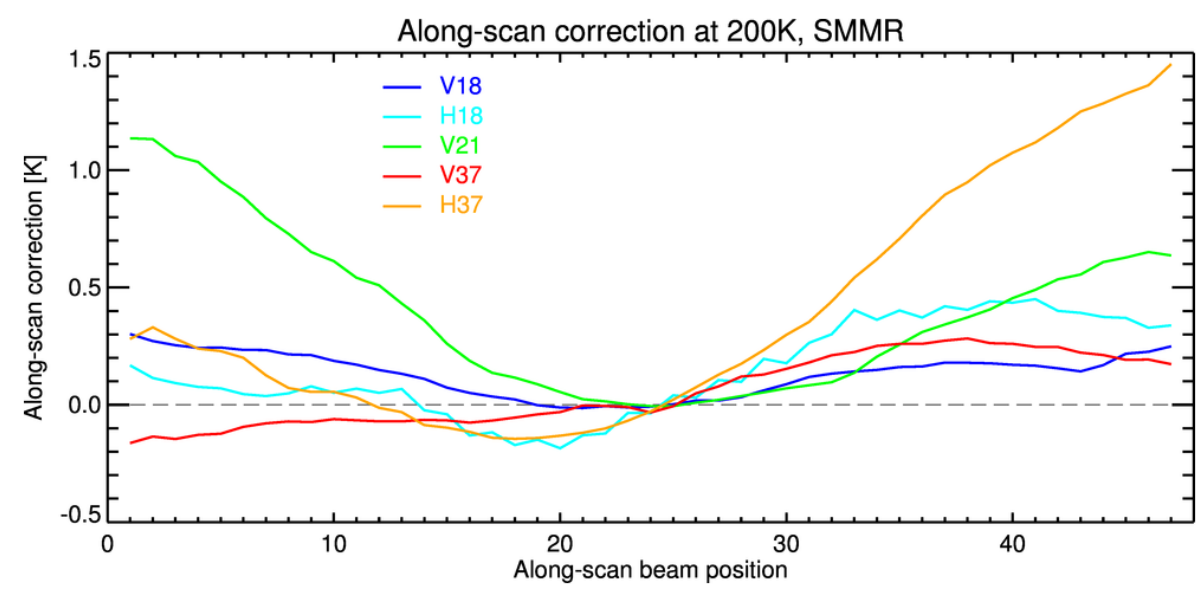

Figure 7. Scan-angle-dependent correction for the SMMR instruments. This image shows the along-scan correction at a common scene temperature of $200 \mathrm{~K}$. Nadir is at position 24. Channel colours are as follows: blue - v18; cyan - h18; green - v21; red - v37; orange - h37.

$G_{p p}\left(\widehat{\boldsymbol{s}_{0}}, \widehat{\boldsymbol{s}}\right)$ and cross-polarized antenna pattern $G_{p q}\left(\widehat{\boldsymbol{s}_{0}}, \widehat{\boldsymbol{s}}\right)$ :

$$
\begin{aligned}
T_{\mathrm{A}_{p}}\left(\widehat{\boldsymbol{s}_{0}}\right) & =\int_{4 \pi}\left[G_{p p}\left(\widehat{\boldsymbol{s}_{0}}, \widehat{\boldsymbol{s}}\right) T_{\mathrm{B}_{p}}(\rho, \widehat{\boldsymbol{s}})\right. \\
& \left.+G_{p q}\left(\widehat{\boldsymbol{s}_{0}}, \widehat{\boldsymbol{s}}\right) T_{\mathrm{B}_{q}}(\rho, \widehat{\boldsymbol{s}})\right] \mathrm{d} \Omega,
\end{aligned}
$$

where $q$ is the polarization orthogonal to $p$.

The measured antenna temperature $T_{\mathrm{A}}$ is therefore directly related to the radiation entering the antenna feedhorn as observed with the effective co- and cross-polarized far-field antenna power patterns. To obtain a correct estimate of the actual scene brightness temperature $T_{\mathrm{B}}$ from the measured $T_{\mathrm{A}}$, it is necessary to correct for the radiation from the crosspolarization (cross-polarization leakage $\chi_{v, h}$ ) and from the cold space (feedhorn spillover loss $\Lambda_{\mathrm{sp}}$ ) (Hollinger et al., 1987). These corrections are generally referred to as antenna pattern correction (APC). In order to minimize systematic uncertainties in the computed brightness temperature data record, the antenna specifications must be carefully characterized before launch.

Introducing $\phi$ as the angle between the antenna polarization vector and the Earth polarization vector with the antenna gain as $\sin ^{2} \phi$ dependence (Njoku et al., 1998) Eq. (18) can be written as

$$
\begin{aligned}
T_{\mathrm{A}_{p}} & =\int_{4 \pi}\left[G_{p p}\left(T_{\mathrm{B}_{p}} \cos ^{2} \phi+T_{\mathrm{B}_{q}} \sin ^{2} \phi\right)\right. \\
& \left.+G_{p q}\left(T_{\mathrm{B}_{q}} \cos ^{2} \phi+T_{\mathrm{B}_{p}} \sin ^{2} \phi\right)\right] \mathrm{d} \Omega .
\end{aligned}
$$

This integral can be subdivided into an Earth-view region $\Omega_{\mathrm{e}}$ and a cold-space region $\Omega_{\mathrm{sp}}$. It is assumed that the coldspace cosmic background $T^{\mathrm{c}}$ is constant at $2.7 \mathrm{~K}$. The coldspace contribution

$\Lambda_{\mathrm{sp}}=\int_{\Omega_{\mathrm{sp}}}\left(G_{p p}+G_{p q}\right) \mathrm{d} \Omega$ is subtracted from the antenna temperature while the antenna gains are renormalized over the Earth-view region to derive the Earth antenna temperature $T^{\prime} \mathrm{A}_{p}$ :

$T^{\prime}{ }_{\mathrm{A} p}=\frac{T_{\mathrm{A}_{p}}-T^{\mathrm{c}} \cdot \Lambda_{\mathrm{sp}}}{1-\Lambda_{\mathrm{sp}}}$.

This is also referred to as spillover correction or space side-lobe correction and is applied for all instruments. The spillover fractions for SMMR are taken from Fu et al. (1988), for SSM/I from Wentz (1991), and for SSMIS from the corresponding ground processing software.

A detailed description of the derivation of the equations to compute the $T_{\mathrm{B}}$ values for each individual sensor is given in Appendix A.

\section{Inter-calibration of sensors}

In order to ensure a homogeneous time series of brightness temperatures from all channels which are available from SMMR across SSM/I to SSMIS, the varying individual instrument characteristics have to be corrected by an intersensor cross calibration of the individual radiometers.

An absolute inter-calibration of the microwave instruments is not possible because there are no absolute reference SI-traceable targets available. The first step for an inter-calibration is therefore to select a suitable reference target and then to transfer this calibration to all other sensors. This inter-sensor calibration method is designed to provide a homogeneous time series with any potential absolute, but unknown, offset observed in the reference brightness temperatures transferred to all other radiometers. The inter-calibration reference selected for the CM SAF FCDR is the SSM/I aboard DMSP F11 (Andersson et al., 2010). In tests with different wind speed algorithms against collocated in situ buoy data, this radiometer exhibits a reliable long-term 
stability. Furthermore, it has a temporal overlap with most of the other SSM/I radiometers which does allow a direct intercalibration. Selecting a reference in the middle of a chain of instruments also minimizes the number of inter-calibration steps needed to harmonize the full constellation. Each intercalibration step introduces a new uncertainty. Selecting a reference at the end of the chain would therefore result in a large uncertainty at the beginning of a climate data record.

The Equator crossing times of the SSMI(S) platforms are not constant but can drift to earlier or later times by up to $3-4 \mathrm{~h}$, depending on the spacecraft. This change must be correctly taken into account in the inter-calibration models (see Sect. 5.2 and 5.3) in order to preserve the natural diurnal variability. However, this difference in observation time adds to the uncertainty of the inter-calibration.

\subsection{SSM/I and SSMIS Earth incidence angle normalization}

The SSM/I scanning system is designed to observe the Earth's surface under a nominal constant Earth incidence angle (EIA) of $53^{\circ}$. A deviation of $1^{\circ}$ from the nominal incidence angle causes $T_{\mathrm{B}}$ changes of up to $2 \mathrm{~K}$ depending on surface and atmospheric conditions. Errors in the geophysical parameters can be on the order of $5 \%$ to $10 \%$ if the variation in the incidence angle is not taken into account (Furhop and Simmer, 1996).

The incidence angle varies mainly due to changes in the altitude of the spacecraft, caused by the eccentricity of the orbit and the oblateness of the Earth. The EIA can also vary across the scan by approximately $0.1^{\circ}$ due to pitch and roll variations in the attitude of the spacecraft. For example, the altitude of the F13 varies along the orbit between 840 and $880 \mathrm{~km}$, corresponding to a variation of about $0.3^{\circ}$ in EIA. Also, a seasonal variation is apparent with a period of about $122 \mathrm{~d}$. This periodic change is caused by the precession of the argument of perigee due to the Earth's oblateness. The strongest variation is found for the F10 with about $1^{\circ}$ due to an orbit with higher ellipticity. Not only the variation but also the mean EIA differ across the radiometers and even varies for different channels of an SSMIS instrument because the individual feedhorns are slightly differently aligned.

The varying EIA must be taken into account either explicitly by a surface emissivity model in a geophysical retrieval scheme or by normalizing the $T_{\mathrm{B}}$ values to a nominal EIA value. This is not only the case when geophysical parameters are derived but also an important prerequisite for the inter-sensor calibration procedure as two sensors observe the same area at the same time but at different EIAs. The normalization is made available as an additional offset variable in the CM SAF FCDR data files, which can be added to the inter-calibrated $T_{\mathrm{B}}$ values. The offsets are computed using the method described by Furhop and Simmer (1996) for water-type FOVs only. This method uses the observed $T_{\mathrm{B}}$ vector to estimate the slope of the $T_{\mathrm{B}}$ change with respect to the incidence angle $\theta$ :

$\mathrm{sl}=\frac{\delta T_{\mathrm{B}}}{\delta \theta}$.

The $T_{\mathrm{B}}$ values are then normalized in an iterative procedure:

$\mathrm{sl}=a_{0}+\sum_{i} a_{i} \cdot T_{\mathrm{B}}\left(\theta=53^{\circ}\right)$

$T_{\mathrm{B}}(\theta)=T_{\mathrm{B}}\left(\theta=53^{\circ}\right)+\mathrm{sl} \cdot \Delta \theta$,

where $a_{i}$ denotes the regression coefficients of the Furhop and Simmer model for the $T_{\mathrm{B}}$ values observed at 19, 22, and $37 \mathrm{GHz}$. The slope "sl" depends on the atmospheric and surface conditions. The procedure is only applicable over ocean because the regression coefficients are determined for this surface type. The final normalization offset $\Delta T_{\mathrm{B}}^{\text {eia }}$ is then defined as

$\Delta T_{\mathrm{B}}^{\text {eia }}=T_{\mathrm{B}}\left(\theta=53^{\circ}\right)-T_{\mathrm{B}}(\theta)$.

\subsection{SSM/I and SSMIS inter-calibration model}

The SSM/I and SSMIS sensors are spectrally inter-calibrated and thus provide a seamless data record of the SSM/I frequencies at 19,22, and $37 \mathrm{GHz}$. The $85 \mathrm{GHz}$ channels on SSM/I are replaced with $91 \mathrm{GHz}$ channels on SSMIS. In order to allow the continued usage of existing algorithms, synthetic $85 \mathrm{GHz} T_{\mathrm{B}}$ values are estimated from the $91 \mathrm{GHz} T_{\mathrm{B}}$ values, inter-calibrated to the SSM/I time series and provided in the FCDR data files. Further details can be found in the corresponding algorithm theoretical basis document (ATBD) (Fennig et al., 2017). The SSM/I instruments aboard F08 and F15, which do not have a temporal overlap with F11, are calibrated to the corrected F10 and F13 radiometers, employed as transfer standards respectively. Also, the SSMIS sensors do not have a temporal overlap with F11. Here SSMIS ${ }_{\mathrm{F} 16}$ is inter-calibrated to the corrected $\mathrm{SSM} / \mathrm{I}_{\mathrm{F} 13}$ radiometer in a first step. Then the corrected SSMIS $\mathrm{F} 16_{6}$ is used as the transfer standard for the SSMIS radiometers aboard F17 and F18. The inter-calibration model is an updated version of the homogenization scheme described in Andersson et al. (2010). The largest contributions to the expected systematic uncertainties, and thus the sources of expected differences, arise from the calibration non-linearity and the imperfect knowledge of the radiometer cross-polarization leakage and spillover loss (see Sect. 6). In order to account for these issues, the inter-calibration model was designed to correct for observed inter-sensor $T_{\mathrm{B}}$ differences as a function of scene temperature and the scene polarization difference $\Delta_{p q}=T_{\mathrm{B}_{v}}-T_{\mathrm{B}_{h}}$. The model comprises an offset term $a$, a scene-dependent linear correction term $b$, a polarization difference term $c$, and a non-linearity term $d$. The non-linearity correction depends on the temperature difference of the observed scene temperature from both calibration targets and is applied as a part of the radiometric calibration on the antenna temperatures $T_{\mathrm{A}}$ :

$T_{\mathrm{A}}^{\#}=T_{\mathrm{A}}+d \cdot\left(T_{\mathrm{A}}-\left\langle T^{\mathrm{h}}\right\rangle\right) \cdot\left(T_{\mathrm{A}}-T^{\mathrm{c}}\right)$, 
where $T_{\mathrm{A}}^{\#}$ is the antenna temperature with non-linearity corrections applied, $T^{\mathrm{h}}$ is the warm target temperature, and $T^{\mathrm{c}}$ is the cold-space cosmic background temperature. The other inter-calibration terms are applied to the brightness temperatures (after application of APC, see Sect. 4) to finally derive the inter-calibrated brightness temperature $T_{\mathrm{B}}^{\text {ic }}$ :

$T_{\mathrm{B}}^{\#}=\operatorname{APC}\left(T_{\mathrm{A}}^{\#}\right)$,

$T_{\mathrm{B}}^{\mathrm{ic}}=a+b \cdot T_{\mathrm{B}}^{\#}+c \cdot \Delta_{p q}$.

The reason to split the inter-sensor calibration procedure is to keep the corrections as logical enhancements to specific processing steps in the calibration and the antenna pattern correction.

The individual inter-sensor calibration terms are determined by fitting the inter-calibration model Eqs. (25) and (26) using match-up data sets of the radiometers compiled from the final $T_{\mathrm{B}}$ values on the lower $\operatorname{SSMI}(\mathrm{S})$ resolutions, i.e. after application of antenna pattern matching to the high-frequency channels (see Sect. 3.3). The final $T_{\mathrm{B}}$ values include all corrections as described in Sect. 3 plus the incidence angle normalization offset $\Delta T_{\mathrm{B}}^{\text {eia }}$ for oceanic scenes (see Sect. 5.1) in order to derive the inter-calibration coefficients at a fixed nominal EIA.

The data sets used for the inter-calibration are constructed from absolute $T_{\mathrm{B}}$ values of oceanic, sea ice, and cold land scenes plus the polarization differences over all surface types. This selection of $T_{\mathrm{B}}$ values uses the Earth as an inter-calibration transfer target, with the relative cold ocean as a vicarious cold reference and the warm land (via the polarization difference) as a vicarious warm reference inter-calibration target. The polarization difference must be preserved during the inter-calibration. The inter-calibration scale factors and offset terms for vertical and horizontal polarization are therefore not independent. The polarization difference term can thus be used as a constraint in the intercalibration procedure across all surface types. This method also ensures that the complete natural variability of the observations is taken into account, and thus extrapolation is limited to extreme values which are damped due to the averaging process.

The brightness temperatures from each channel and instrument are binned into a daily global $1^{\circ}$ equal-angle grid, separately for morning and evening orbits. For each radiometer a match-up data set with the inter-calibration reference or the selected (and corrected) transfer standard is then compiled from the collocated gridded mean normalized $T_{\mathrm{B}}$ values for the entire overlap period. Only grid cells with morning and evening (local time) observations are selected and averaged to daily mean observations in order to minimize the influence of diurnal cycle variations, assuming sinusoidal characteristics. The polarization differences are much less affected by the diurnal cycle as the vertical and horizontal polarization at the same frequency exhibits similar diurnal variations. These daily mean observations are finally averaged to pro- duce monthly mean $T_{\mathrm{B}}$ maps. The monthly mean $1^{\circ}$ grid cells are treated as individual independent observations and the calibration coefficients in Eqs. (25) and (26) are fitted by minimizing the observed temperature differences to the selected target instrument.

Due to the stable in-orbit calibration of the SSMI(S) sensors, the coefficients are considered to be constant during the lifetime of a radiometer and are used to homogenize the measurements of the different instruments. The inter-calibration offsets $\Delta T_{\mathrm{B}}^{\text {ic }}$ to the $T_{\mathrm{B}}$ values are assumed to be valid over all surface types and computed during the final processing for each FOV and then separately archived in the FCDR data files:

$\Delta T_{\mathrm{B}}^{\mathrm{ic}}=T_{\mathrm{B}}^{\mathrm{ic}}-T_{\mathrm{B}}$.

By keeping the incidence angle normalization offsets $\Delta T_{\mathrm{B}}^{\text {eia }}$ and the inter-calibration offsets $\Delta T_{\mathrm{B}}^{\mathrm{ic}}$ separately from the $T_{\mathrm{B}}$ values themselves, each user has the freedom to choose whatever correction or combination is adequate for their application. The inter-calibration can be applied independently of the EIA correction with an error of $<0.1 \mathrm{~K}$.

\subsection{SMMR inter-calibration model}

The overlap period of SMMR and SSM// $\mathrm{I}_{\mathrm{F} 08}$ is just about 1 month. This is not a sufficient record length to directly derive stable inter-calibration coefficients as seasonal or other multi-monthly variations can not be accounted for. It is therefore not possible to use the statistical methods developed for the SSMI(S) inter-calibration. Instead, a double-difference technique using modelled brightness temperatures from reanalysis profiles is employed here following the approach from Sapiano et al. (2013).

It is important to keep in mind that this inter-calibration of the SMMR is not intended to completely remove all observed differences. Instead the measured SMMR $T_{\mathrm{B}}$ values are corrected to be physically consistent with corresponding SSM/I $T_{\mathrm{B}}$ values. This means small differences due to different nominal channel centre frequencies (e.g. $18 \mathrm{GHz}$ vs. $19 \mathrm{GHz}$ ) and different bandwidths are to be expected. Also, the different nominal EIAs of the measurements $\left(50^{\circ}\right.$ vs. $\left.53^{\circ}\right)$ will result in overall and time-varying differences. These must be accounted for not only during the inter-calibration procedure in order to derive physically consistent inter-calibration coefficients, but also later on in the application. This can be done either directly in the retrieval algorithm or indirectly by normalizing the observed $T_{\mathrm{B}}$ to a nominal frequency and EIA.

Using a reanalysis as a transfer standard has advantages but also limitations. Firstly, the most important requirement here is the temporal stability of the reanalysis. This is the basic underlying assumption to allow a transfer of an observed bias from the SSM/I time period to the SMMR time period. Secondly, the uncertainties in the applied radiative transfer model and the surface emissivity model should be well be- 
haved in order to cancel out in the double-difference technique.

While using modelled $T_{\mathrm{B}}$ values for the inter-calibration, the EIA dependence of the $T_{\mathrm{B}}$ values and the frequency shifts are explicitly accounted for. Also, the different Equator overpass times of Nimbus-7 (00:00) and DMSP F08 (06:00) are considered by the temporal collocation criterion. However, a limitation is the large uncertainty of surface emissivity models over land, ice, and snow-covered surfaces due to unknown surface emissivities. Also, the strong diurnal cycle over land is not resolved by the reanalysis time steps. Additionally, rainy and cloudy scenes increase the uncertainty of the radiative transfer model due to scattering effects of the water droplets and ice crystals. In order to minimize these influences, only cloud-free and lightly cloudy scenes over water surfaces are selected for the inter-calibration match-up data sets.

The profile and surface data used here are taken from the ERA-20C reanalysis (Poli et al., 2013). Brightness temperatures at the top of the atmosphere are calculated with RTTOV 11.2 (Saunders et al., 2013) and the surface emissivity with FASTEM 6 (Meunier et al., 2014). Profiles from ERA-20C are available every $3 \mathrm{~h}$, resulting in maximum time differences of $90 \mathrm{~min}$ between observed and simulated $T_{\mathrm{B}}$ values. The simulations are carried out for the entire time period covered by the non-inter-calibrated SMMR and the SSM/I FCDR for F08. In order to reduce the uncertainty in the observed differences, the match-up data are gridded into daily global $1^{\circ}$ equal-angle grids, separately for ascending and descending orbits. As ERA-20C does not include satellite observations, this likely assures that the $T_{\mathrm{B}}$ values in both independent data records are stable over the selected time period.

No significant trends can be observed in the global monthly mean differences between simulated and observed $T_{\mathrm{B}}$ values for the $\mathrm{SSM} / \mathrm{I}_{\mathrm{F} 08}$. Morning and evening $\mathrm{SSM} / \mathrm{I}$ $T_{\mathrm{B}}$ anomalies depict nearly identical temporal variations and agree to within $0.2 \mathrm{~K}$ in the mean value (not shown). Only the channel at $37 \mathrm{GHz}$, h-pol shows a larger difference with a value of $0.4 \mathrm{~K}$ between ascending and descending orbits. This also underlines the stability and quality of the SSM/I FCDR. The SMMR anomalies behave differently. Generally they are stable over time despite the last months of 1987 when the differences become smaller in all channels. The $21 \mathrm{GHz}$, v-pol channel seems to be affected by a small upward drift towards the end of its lifetime (1983-1985). These trends are most likely caused by the correction of the longterm trend in the level 1B data record (see Sect. 3.6). As this correction was derived from a polynomial fit of the absolute global mean values, existing natural climate trends are not correctly taken into account. Also, seasonal variations in the ascending and descending orbits can be observed, which do not agree. Phase shifts in the anomalies are visible for the channels at $18 \mathrm{GHz}$, v-pol; $21 \mathrm{GHz}$, v-pol; and both channels at $37 \mathrm{GHz}$. The amplitude of the variations decreases with increasing frequency from about 2 to $0.5 \mathrm{~K}$.

It was not possible to identify the root cause for these temporal variations and offsets. The magnitude of the observed $T_{\mathrm{B}}$ anomalies and their overall decrease with frequency might be caused by unresolved variations in the EIA. This can be due to either errors in the satellite attitude control or deficiencies in the applied short-term, orbit-angledependent correction in the level $1 \mathrm{~B}$ data record. These shortterm corrections were derived by forcing ascending-minusdescending $T_{\mathrm{B}}$ differences to be zero without accounting for the different EIA values at ascending and descending paths or diurnal variations. As this level $1 \mathrm{~B}$ correction is based on look-up tables, which are not available, it can not be reverted. Hence, it is not possible to separate the effects and identify the physical reasons for the observed seasonal variations.

The inter-calibration model employed for the CM SAF SMMR data record is adapted from the absolute calibration correction as applied in the level 1B data processing. This correction is a scene-dependent correction to a modelled background acting as a cold vicarious calibration target while keeping the warm calibration target at the instrument warm load reference temperature. Selecting a simple $T_{\mathrm{B}}$ inter-calibration offset derived at the cold end would result in scene-dependent biases at warm scene temperatures. The cold target for the FCDR inter-calibration is now defined as the inter-calibrated $\mathrm{SSM} / \mathrm{I}_{\mathrm{F} 08} T_{\mathrm{B}}$ acting as a transfer target to the $\mathrm{SSM} / \mathrm{I}_{F 11}$ reference sensor. Keeping the nomenclature from Eq. (17) (Njoku et al., 1998), a linear correction is then defined as

$T_{\mathrm{B}}^{\mathrm{ic}}=a+c \cdot T_{\mathrm{B}}$,

where $T_{\mathrm{B}}$ is the observed SMMR brightness temperature, $T_{\mathrm{B}}^{\mathrm{ic}}$ is the inter-calibrated value, and $a$ and $c$ are the intercalibration coefficients. The constraints can be defined as

$$
\begin{aligned}
& T_{\mathrm{B}}^{\mathrm{c}}=T_{\mathrm{B}}^{\mathrm{o}}+\left(T_{\mathrm{B}}^{\mathrm{m}}-T_{\mathrm{B}}^{\mathrm{o}}\right)-\left(T_{\mathrm{B}}^{\# \mathrm{~m}}-T_{\mathrm{B}}^{\# \mathrm{o}}\right), \\
& T_{\mathrm{B}}^{\mathrm{c}}=a+c \cdot T_{\mathrm{B}}^{\mathrm{o}}, \\
& I^{\prime}=a+c \cdot I^{\prime},
\end{aligned}
$$

where $T_{\mathrm{B}}^{\mathrm{c}}$ is the mean corrected SMMR brightness temperature, $T_{\mathrm{B}}^{\mathrm{O}}$ is the mean observed SMMR brightness temperature, and $T_{\mathrm{B}}^{\mathrm{m}}$ is the modelled brightness temperature. $\left(T_{\mathrm{B}}^{\mathrm{m}}-T_{\mathrm{B}}^{\mathrm{o}}\right)$ is the mean SMMR difference between modelled and observed temperatures, $\left(T_{\mathrm{B}}^{\# \mathrm{~m}}-T_{\mathrm{B}}^{\# \mathrm{o}}\right)$ is the corresponding mean SSM/I brightness temperature difference, and $I^{\prime}$ is the normalized warm load reference brightness, which shall not change. These equations are solved for the intercalibration coefficients $a$ and $c$ :

$$
\begin{aligned}
& a=I^{\prime} \cdot \frac{T_{\mathrm{B}}^{\mathrm{c}}-T_{\mathrm{B}}^{\mathrm{o}}}{I^{\prime}-T_{\mathrm{B}}^{\mathrm{o}}}, \\
& c=\frac{I^{\prime}-T_{\mathrm{B}}^{\mathrm{c}}}{I^{\prime}-T_{\mathrm{B}}^{\mathrm{o}}} .
\end{aligned}
$$


The coefficients $a$ and $c$ depend on the actual warm load equivalent brightness $I^{\prime}$ and are updated online for each new set of calibration data. The inter-calibration offsets $\Delta T^{\text {ic }}$ to the observed $T_{\mathrm{B}}$ values are assumed to be valid over all surface types and computed during the final processing for each FOV and then archived in the SMMR FCDR data files.

\section{Uncertainty estimates}

It is not possible to specify a complete systematic uncertainty estimate for the observed $T_{\mathrm{B}}$ values because of a general limitation to trace observed systematic differences back to SI standards for microwave observations (Hollinger et al., 1990). As there are no absolute reference observations available to validate the absolute radiometer calibration, each instrument must be treated as a unique observing system. This means the measured brightness temperatures can only be compared against similar observations, for example aircraft measurements with an SSM/I or SSMIS simulator, or against simulated $T_{\mathrm{B}}$ values computed with a radiative transfer model. In this case, the exact channel specifications must be known. But the radiative transfer model and the employed surface emissivity model will also have random and systematic uncertainty sources, which have to be properly characterized. The observed and the modelled $T_{\mathrm{B}}$ values can then be compared to test whether they agree within their defined total uncertainties.

Both methods have been used to evaluate the absolute calibration of SSM/I during the F08 instrument cal/val programme (Hollinger et al., 1990). The conclusion from these evaluation experiments is that the absolute calibration shows no significant systematic bias and may be better than the uncertainty of its determination of $\pm 3 \mathrm{~K}$.

The total expected uncertainty of a quantity can be divided into two components: a systematic uncertainty and a random uncertainty. While the random uncertainty of the $T_{\mathrm{B}}$ values can be determined on-orbit statistically from the instrument measurements used to calibrate the radiometer, the total systematic uncertainty must be identified during prelaunch ground measurements, following the ISO Guide to the Expression of Uncertainty in Measurement (ISO GUM, JCGM 100:2008, 2008).

\subsection{Random uncertainty}

The random uncertainty of a radiometer is usually expressed as a noise-equivalent temperature $\mathrm{Ne} \Delta \mathrm{T}$, which is the standard uncertainty of an individual measurement. This uncertainty is defined as the standard deviation of the measurement referenced to the energy of the radiation incident on the antenna. This noise can be estimated using the on-orbit calibration measurements and the calibration equation. Applying standard error propagation to the SSMI(S) radiometric calibration Eqs. (2) and (3) and dropping the averaging symbols here to increase readability,

$\mathrm{Ne} \Delta \mathrm{T}^{2}=U\left(T^{\mathrm{h}}\right)^{2}+U\left(C^{\mathrm{h}}\right)^{2}+U\left(C^{\mathrm{c}}\right)^{2}+U\left(C^{\mathrm{e}}\right)^{2}$,

with the following standard uncertainties contributing to the combined total standard variability:

a. standard uncertainty due to noise in the smoothed warm target temperature readings $\sigma_{T^{\mathrm{h}}}$,

$$
U\left(T^{\mathrm{h}}\right)=\frac{\delta T_{\mathrm{A}}}{\delta T^{\mathrm{h}}} \cdot \sigma_{T^{\mathrm{h}}}=\frac{C^{\mathrm{e}}-C^{\mathrm{c}}}{C^{\mathrm{h}}-C^{\mathrm{c}}} \cdot \sigma_{T^{\mathrm{h}}},
$$

b. standard uncertainty due to noise in the smoothed warm calibration counts $\sigma_{C^{\mathrm{h}}}$,

$$
U\left(C^{\mathrm{h}}\right)=\frac{\delta T_{\mathrm{A}}}{\delta C^{\mathrm{h}}} \cdot \sigma_{C^{\mathrm{h}}}=\frac{\left(T^{\mathrm{h}}-T^{\mathrm{c}}\right) \cdot\left(C^{\mathrm{c}}-C^{\mathrm{e}}\right)}{\left(C^{\mathrm{h}}-C^{\mathrm{c}}\right)^{2}} \cdot \sigma_{C^{\mathrm{h}}},
$$

c. standard uncertainty due to noise in the smoothed cold calibration counts $\sigma_{C^{c}}$,

$$
U\left(C^{\mathrm{c}}\right)=\frac{\delta T_{\mathrm{A}}}{\delta C^{\mathrm{c}}} \cdot \sigma_{C^{\mathrm{c}}}=\frac{\left(T^{\mathrm{h}}-T^{\mathrm{c}}\right) \cdot\left(C^{\mathrm{e}}-C^{\mathrm{h}}\right)}{\left(C^{\mathrm{h}}-C^{\mathrm{c}}\right)^{2}} \cdot \sigma_{C^{\mathrm{c}}},
$$

d. standard uncertainty due to noise in the Earth counts $\sigma_{C^{e}}$,

$$
U\left(C^{\mathrm{e}}\right)=\frac{\delta T_{\mathrm{A}}}{\delta C^{\mathrm{e}}} \cdot \sigma_{C^{\mathrm{e}}}=\frac{T^{\mathrm{h}}-T^{\mathrm{c}}}{C^{\mathrm{h}}-C^{\mathrm{c}}} \cdot \sigma_{C^{\mathrm{e}}} .
$$

The standard deviations of the smoothed cold counts $\sigma_{C^{c}}$, smoothed warm counts $\sigma_{C^{\mathrm{h}}}$, and smoothed temperature readings $\sigma_{T^{\mathrm{h}}}$ are estimated from all available valid measurements on a daily basis. The daily mean noise-equivalent temperature $\mathrm{Ne} \Delta \mathrm{T}$ for each channel can then be estimated while viewing the warm target, i.e. replacing $C^{\mathrm{e}}$ with $C^{\mathrm{h}}$, and applying the standard deviation of the individual warm counts. As the variability of the warm counts is generally larger than the variability of the cold counts, this value is a valid estimate for the maximum expected $\mathrm{Ne} \Delta \mathrm{T}$. Due to bandwidth limitations, the cold and warm load readings of the SSMIS instruments are only available as a mean value over a predefined number of beam positions (scan line mean). This number differs between the instruments and also changes over time. The variance of the individual readings is therefore estimated from the variance of the scan line mean values by multiplying these with the number of averaged beam positions valid at that observation time.

The term with the largest contribution to the total standard uncertainty is the uncertainty due to noise in the Earth counts $U\left(C^{\mathrm{e}}\right)$ with about $98 \%$. The contribution from the uncertainty in the temperature readings can be neglected while the uncertainty in the cold and warm calibration target readings contribute the remaining $2 \%$. The across-scan smoothing applied to the scan line mean values (Eq. 5) reduces $\mathrm{Ne} \Delta \mathrm{T}$ by 
approximately $10 \%$. While reducing the noise of the individual observations, the across-scan averaging introduces a small error covariance over the smoothing kernel width.

$\mathrm{The} \mathrm{Ne} \Delta \mathrm{T}$ for SMMR is derived from the corresponding radiometric calibration Eq. (7):

$$
\begin{aligned}
\mathrm{Ne} \Delta \mathrm{T}^{2} & =U(I)^{2}+U(S)^{2}+U\left(C^{\mathrm{h}}\right)^{2} \\
& +U\left(C^{\mathrm{c}}\right)^{2}+U\left(C^{\mathrm{e}}\right)^{2},
\end{aligned}
$$

with the following standard uncertainties contributing to the combined standard variability:

a. standard uncertainty due to noise in the calibration brightness $I$,

$$
U(I)=\frac{\delta T_{\mathrm{A}}}{\delta I} \cdot \sigma_{I}=S \cdot \frac{C^{\mathrm{e}}-C^{\mathrm{h}}}{C^{\mathrm{c}}-C^{\mathrm{h}}} \cdot \sigma_{I},
$$

b. standard uncertainty due to noise in the calibration slope coefficient $S$,

$$
U(S)=\frac{\delta T_{\mathrm{A}}}{\delta S} \cdot \sigma_{S}=\frac{C^{\mathrm{e}}-C^{\mathrm{h}}}{C^{\mathrm{c}}-C^{\mathrm{h}}} \cdot \sigma_{S},
$$

c. standard uncertainty due to noise in the averaged warm counts $C^{\mathrm{h}}$,

$$
U\left(C^{\mathrm{h}}\right)=\frac{\delta T_{\mathrm{A}}}{\delta C^{\mathrm{h}}} \cdot \sigma_{C^{\mathrm{h}}}=S \cdot \frac{C^{\mathrm{e}}-C^{\mathrm{c}}}{\left(C^{\mathrm{c}}-C^{\mathrm{h}}\right)^{2}} \cdot \sigma_{C^{\mathrm{h}}},
$$

d. standard uncertainty due to noise in the averaged cold counts $C^{\mathrm{c}}$,

$$
U\left(C^{\mathrm{c}}\right)=\frac{\delta T_{\mathrm{A}}}{\delta C^{\mathrm{c}}} \cdot \sigma_{C^{\mathrm{c}}}=S \cdot \frac{C^{\mathrm{h}}-C^{\mathrm{e}}}{\left(C^{\mathrm{c}}-C^{\mathrm{h}}\right)^{2}} \cdot \sigma_{C^{\mathrm{c}}},
$$

e. standard uncertainty due to noise in the Earth counts $C^{\mathrm{e}}$,

$$
U\left(C^{\mathrm{e}}\right)=\frac{\delta T_{\mathrm{A}}}{\delta C^{\mathrm{e}}} \cdot \sigma_{C^{\mathrm{e}}}=S \cdot \frac{1}{C^{\mathrm{c}}-C^{\mathrm{h}}} \cdot \sigma_{C^{\mathrm{e}}}
$$

The contributing standard deviations are estimated on a daily basis similar to SSMI(S) but using the cold target because the variability of the SMMR cold counts is larger than the variability of the warm count. All derived variances and $\mathrm{Ne} \Delta \mathrm{T}$ values are archived in the FCDR data files as daily mean values in order to provide full traceability.

\subsection{Systematic uncertainty}

The total systematic radiometer calibration uncertainty budget can be composed of the following contributors: (1) warm load reference uncertainty, (2) cosmic background reference uncertainty, (3) radiometer/calibration non-linearity, (4) radiative coupling $\varepsilon$ uncertainty, (5) uncertainty in the APC coefficients, (6) reflector emissivity, and (7) along-scan crosspolarization mixing. Evaluation results for the SSM/I radiometric calibration have been reported by Hollinger et al. (1989) and Colton and Poe (1999), for SSMIS by Kunkee et al. (2008c), and for SMMR by Njoku et al. (1998). These results are discussed in the following sections and uncertainty values for each contributing term are estimated. The derived uncertainties are summarized in Table 1.

\subsubsection{Warm load reference uncertainty}

The calibration uncertainty of the warm load was determined pre-launch for $\mathrm{SSM} / \mathrm{I}_{\mathrm{F} 08}$ by comparison with a variableprecision calibration reference target over a range of 100 to $375 \mathrm{~K}$ during thermal vacuum calibration. These tests show no systematic calibration error (Hollinger et al., 1989). Kunkee et al. (2008c) refer to an absolute uncertainty of $0.1 \mathrm{~K}$ for SSMIS. However, no information on pre-launch measurements of the temperature sensors is available for SMMR. A common value of $0.1 \mathrm{~K}$ is employed for all instruments.

\subsubsection{Cosmic background reference uncertainty}

An analysis of the calibration reflector antenna patterns, when the SSM/I is in the calibration position, reveals that the reception of Earth and spacecraft radiation is extremely small (less than a few tenths of a degree). Thus it is believed that the SSM/I calibration reflector provides a clear view of the cosmic background to the feedhorn and hence provides a highly accurate black-body calibration reference (Hollinger et al., 1989). However, this is not the case during phases when parts of the moon are in the field of view of the reflector (see Sect. 3.6.2). These short-lived intrusion events can cause an underestimation of the true scene temperature of up to $0.5 \mathrm{~K}$, depending on the channel frequency and scene temperature. As the SSMIS employs the same cold calibration method, the same problem arises and the same maximum bias is assumed.

The SMMR suffers from periodic sunlight intrusion events into the cold-sky calibration horns. This usually occurs once during each orbit over the southern polar region. These regions are not used in the calibration. Instead the cold counts are linearly interpolated between the last valid values (Njoku et al., 1998). Apart from these events, it is believed that the SMMR calibration horns provide a clear view of the cosmic background.

To account for these short-term SSMI(S) intrusions and the uncertainties in the SMMR calibration interpolation, a mean uncertainty of $0.1 \mathrm{~K}$ is assumed for all instruments, keeping in mind that only small parts of the orbit are affected. 
Table 1. Summary of estimated systematic uncertainty source contributions.

\begin{tabular}{lrr}
\hline Systematic uncertainty source & Systematic uncertainty range $\Delta a(\mathrm{~K})$ & Standard uncertainty $u(\mathrm{~K})$ \\
\hline Warm load reference & - & 0.10 \\
Cosmic background reference & - & 0.10 \\
Calibration non-linearity SSMI(S) & $0.25-0.70$ & $0.15-0.40$ \\
Calibration non-linearity SMMR & - & 0.10 \\
Radiative coupling & $0.10-0.40$ & $0.06-0.25$ \\
Cross-polarization & $0.15-0.30$ & $0.10-0.20$ \\
Feedhorn spillover & $1.00-1.50$ & $0.60-0.90$ \\
Reflector emissivity SSMIS & 0.60 & 0.40 \\
Polarization mixing SMMR & $0.20-0.50$ & $0.10-0.30$ \\
Total SMMR & & $0.40-1.00$ \\
Total SSMIS & & $0.70-1.10$ \\
\hline
\end{tabular}

\subsubsection{Radiometer/calibration non-linearity}

Non-linearities in the calibration measurements and in the radiometer receiver may be expected. This can impact the radiometric calibration when a two-point linear calibration equation is applied. At observation temperatures equal to the calibration reference targets, the calibration uncertainty is the accuracy of the reference, which does not show a systematic uncertainty (see above). At intermediate temperatures, radiometer non-linearity and calibration reference temperature uncertainty contribute to the total uncertainty with the uncertainty weighted according to the difference between the observed scene and the calibration references. To test the radiometer absolute calibration, preflight thermal vacuum tests have been conducted for SSM/I (Colton and Poe, 1999). Calibration cycles were run for three different sensor temperatures: cold $\left(0^{\circ} \mathrm{C}\right)$, ambient $\left(28^{\circ} \mathrm{C}\right)$, and hot $\left(38^{\circ} \mathrm{C}\right)$. For the on-orbit expected operating range the calibration errors are typically -1.4 to $0.5 \mathrm{~K}$ (Colton and Poe, 1999) and hence within the instrument specifications. The corresponding graphs in Colton and Poe (1999) point to a scenetemperature-dependent bias with a larger underestimation for colder scene temperatures. However, the measurements are believed to contain artefacts due to the actual test setup. The on-orbit calibration is expected to perform better than the pre-launch calibration tests indicate (Colton and Poe, 1999).

To test the SSMIS radiometer absolute calibration, thermal vacuum tests have been conducted for all SSMIS instruments, with all meeting the accuracy requirement of $1 \mathrm{~K}$ (Kunkee et al., 2008b). However, a scene-temperaturedependent offset is depicted in Fig. 6a in Kunkee et al. (2008b). Without more details being available, the same values as for SSM/I are used.

Thermal vacuum tests have also been conducted for the SMMR instrument at the Jet Propulsion Laboratory (JPL) for temperature levels ranging from 77 to $320 \mathrm{~K}$ (Gloersen, 1987). Within the observed radiometer switch temperature range of $\pm 2 \mathrm{~K}$, the differences between linear and quadratic calibration equations are found to be within $0.1 \mathrm{~K}$ for all channels.

\subsubsection{Radiative coupling $\varepsilon$ uncertainty}

The radiative coupling coefficient $\varepsilon$ was originally determined for $\mathrm{SSM} / \mathrm{I}_{\mathrm{F} 08}$ and has been used unchanged in the computation of the antenna temperatures (Eq. 6) since then. The correction due to this factor depends on the temperature difference between the warm load and the forward radiator plate. This correction is typically of the order of $0.1 \mathrm{~K}$ for an expected temperature difference of $10 \mathrm{~K}$. However, some instruments show a very strong seasonal variation in the temperature difference reaching $40 \mathrm{~K}$. A relative uncertainty of $1 \%$ in $\varepsilon$ can then lead to a bias of $0.4 \mathrm{~K}$ in the scene brightness temperature.

\subsubsection{Uncertainty in the APC coefficients}

During the ground instrument calibration, a radiometric characterization of the antenna properties is also performed. The performance was established from analyses of the antenna gain function as measured on an antenna range from which, amongst others, feedhorn spillover and cross-polarization loss were determined. The estimated absolute accuracy of the feedhorn spillover is $0.3 \%-0.5 \%$ and the relative crosspolarization accuracy is of the order of 5\%-10\% (Colton and Poe, 1999). The uncertainty in the spillover translates to a scene-dependent uncertainty of the order of about 1$1.5 \mathrm{~K}$ at a $300 \mathrm{~K}$ scene temperature. The uncertainty in the cross-polarization accuracy results in an uncertainty, depending on the scene polarization difference, of the order of 0.15 $0.3 \mathrm{~K}$ for a scene with a $50 \mathrm{~K}$ difference between vertical and horizontal polarization. These values are also employed for SMMR and SSMIS.

\subsubsection{SSMIS reflector emissivity}

The emissive reflector problem is specific for the SSMIS. It leads to an additional systematic uncertainty which de- 
pends on the emissivity of the reflector (see Sect. 3.6.7). The emissivity itself is a function of the channel frequency and the temperature difference between the reflector and the observed target. Thus it is not only scene dependent but also a function of the orbit position when the reflector is heated by the solar radiation and cools in the Earth shadow. The strongest errors are to be expected when the spacecraft leaves the Earth's shadow with the reflector temperature increasing by about $80 \mathrm{~K}$ and at low scene temperatures. With the reflector temperature reaching about $300 \mathrm{~K}$ and the lowest scene temperatures around $150 \mathrm{~K}$, a maximum difference of about $150 \mathrm{~K}$ can be expected. The error due to the emissivity of the reflector can then reach about $13 \mathrm{~K}$ for some of the sounding channels with an estimated emissivity of $\varepsilon_{\text {refl }}=0.09$. The largest error for the SSM/I-like environmental and imager channels appears at $91 \mathrm{GHz}$ for SSMIS $\mathrm{F}_{17}$. With an emissivity of 0.04 at this frequency, this can result in an error of about $6 \mathrm{~K}$. This emissivity-induced error is corrected in the CM SAF FCDR. The expected uncertainty in the determination of the emissivity can be estimated as $\pm 10 \%$, which then translates to a maximum remaining error of $\pm 0.6 \mathrm{~K}$.

\subsubsection{Along-scan cross-polarization mixing}

This systematic uncertainty source is specific for the SMMR as a result of the instrument design. The antenna system consists of a scanning parabolic reflector and a fixed multispectral feedhorn. This leads to a scan-angle-dependent polarization mixing, which is corrected for when the antenna temperature is converted to the $T_{\mathrm{B}}$. This method was developed after launch by Gloersen (1987). Njoku et al. (1998) adopted this method and report the uncertainty of the fitted correction coefficients in the level 1B data record to be between 0.046 and $0.157 \mathrm{~K}$ with the remaining along-scan biases ranging between 0.2 and $0.5 \mathrm{~K}$.

\section{Total systematic uncertainty}

The individual systematic uncertainty contributions are summarized in Table 1 . If only a maximum range can be estimated, the corresponding standard uncertainty $u_{i}$ is derived from the uncertainty range $y_{i}$ assuming a uniform distribution of the uncertainty within the expected range of variation $\Delta a_{i}=\left|\frac{y_{+}-y_{-}}{2}\right|:$

$u_{i}(y)=\frac{\Delta a_{i}}{\sqrt{3}}$.

The combined total expected standard systematic uncertainty

$u_{c}\left(T_{\mathrm{B}}\right)=\sqrt{\sum_{i} u_{i}^{2}(y)}$

is then of the order of $0.7-1.1 \mathrm{~K}$ for $\operatorname{SSMI}(\mathrm{S})$ and $0.4-1 \mathrm{~K}$ for SMMR, with a significant dependence on the scene temperature due to the non-linearity and spillover terms and a minor dependence on the scene polarization. Overall, the total expected systematic uncertainty of the absolute calibration depends on the frequency, the scene temperature, and the polarization difference. As the random uncertainty scales with the number of observations, the systematic uncertainty has to be accounted for when comparing two climatological means of $T_{\mathrm{B}}$ values from different platforms.

\section{Evaluation}

A full evaluation of the CM SAF FCDR was performed and the detailed results are presented in the corresponding validation report, which is freely available from the DOI landing page https://doi.org/10.5676/EUM_SAF_CM/ FCDR_MWI/V003. The corrected and inter-calibrated $T_{\mathrm{B}}$ values were compared to the uncorrected raw data records (RDRs) and to another SSMI(S) brightness temperature data record from Kummerow et al. (2013). The homogeneity of the data records is tested by comparing against the respective ensemble mean of the available satellites in each data record, and additional statistical values are given for bias, robust standard deviation (RSD), median absolute deviation (MAD), and decadal stability.

Conducting a direct inter-sensor validation for the SMMR, as was done for the $\operatorname{SSMI}(\mathrm{S})$ instruments, is not feasible. The overlap time period is just a month and SMMR data are available just every other day. In the case of the $21 \mathrm{GHz}$ channels, there is no overlap at all as these channels failed in 1985. Also, the channels are not at the exact same centre frequency ( 18 and $21 \mathrm{GHz}$ instead of 19 and $22 \mathrm{GHz}$ ) and the SMMR inter-calibration does not account for a frequency shift. Therefore a common stable reference has to be used for both instruments and the anomaly against this reference needs to be analysed.

\subsection{Inter-sensor differences between SSM/I and SSMIS}

In order to quantify the consistency of the brightness temperatures across the SSMI(S) sensors, a reference has to be established first. As there is no absolute reference available and the operating sensors change over time, the ensemble mean of all available instruments at each month has been selected as the relative reference. This approach simplifies further analysis, as it can be performed for each sensor. The inter-sensor differences are derived by comparing the respective bias values to the ensemble mean. The maximum intersensor difference is the ensemble spread, which characterizes the observation uncertainty.

A global monthly mean bias is the mean systematic offset to another sensor. However, it is also important to quantify the observed regional differences, which are characterized by the dispersion of all gridded monthly mean $T_{\mathrm{B}}$ differences around the global mean. The median of absolute differences (MAD), without correcting for the mean systematic offset (BIAS), is a measure of the total absolute uncertainty. A robust (resilient to outliers) measure of the statistical disper- 
sion of a distribution is the median absolute deviation about the median. Assuming a normal distribution, the expected RSD can be estimated from the median absolute deviation by scaling it with a factor of 1.48 . This scaling factor is needed to make the MAD consistent with a standard deviation of a Gaussian distribution.

For the evaluation the data record has been gridded to equal-angle $1^{\circ}$ monthly mean global fields separately for AM and PM orbits. For this comparison all oceanic and sea-icecovered grid cells are used in order to minimize diurnal cycle variations. To derive the relative instrument differences, an ensemble mean data set has been compiled on a monthly basis for all instruments and channels. The ensemble monthly mean grid box brightness temperature $\left\langle T_{\mathrm{B}}\right\rangle$ for each month $t$ and grid box index $g$ is calculated as the arithmetic mean of the individually gridded monthly mean brightness temperatures $T_{\mathrm{B}}$ from all available instruments $s$ :

$\left\langle T_{\mathrm{B}}\right\rangle(t, g)=\frac{1}{N_{s}} \cdot \sum_{i=1}^{N_{s}} T_{\mathrm{B}}\left(s=s_{i}, t, g\right)$,

with $N_{s}$ as the number of contributing instruments for each grid box and month. The distribution of brightness temperature differences $\Delta T_{\mathrm{B}}$ relative to this ensemble mean,

$\Delta T_{\mathrm{B}}(s, t, g)=T_{\mathrm{B}}(s, t, g)-\left\langle T_{\mathrm{B}}\right\rangle(t, g)$,

is then statistically analysed. Robust statistics are applied, with $M$ as the median of $\Delta T_{\mathrm{B}}$ for each instrument $s$. The following statistical quantities are analysed.

$\operatorname{BIAS}_{s_{i}}=M\left[\Delta T_{\mathrm{B}}\left(t, g, s=s_{i}\right)\right]$

$\operatorname{MAD}_{s_{i}}=M\left[\left|\Delta T_{\mathrm{B}}\left(t, g, s=s_{i}\right)\right|\right]$

$\operatorname{RSD}_{s_{i}}=M\left[\left|\mathrm{BIAS}_{s}-\Delta T_{\mathrm{B}}\left(t, g, s=s_{i}\right)\right|\right] \cdot 1.48$

The inter-sensor bias is derived as the difference between two sensors $\left(\mathrm{BIAS}_{s_{1}}-\mathrm{BIAS}_{s_{2}}\right.$ ).

The decadal stability $t_{\mathrm{D}}$ for each channel and instrument is estimated using a linear model trend, fitted to the time series of monthly anomalies relative to the ensemble mean. The monthly anomalies are defined as the median of the global distribution of differences $\Delta T_{\mathrm{B}}$ at each monthly time step $t$ :

$\overline{\Delta T_{\mathrm{B}, s_{i}}}(t)=M\left[\Delta T_{\mathrm{B}}\left(g, s=s_{i}\right)\right](t)$.

A simple model with a linear decadal trend $t_{\mathrm{D}}$ in kelvin per decade can then be defined as

$\overline{\Delta T_{\mathrm{B}, s_{i}}}(t)=\overline{\Delta T_{\mathrm{B}, s_{i}}}(t=0)+\frac{t_{\mathrm{D}}}{120} \cdot t+\varepsilon(t)$,

with $\varepsilon(t)$ representing the fraction of the monthly anomalies not explained by the linear approximation. The linear model terms (offset and trend) are found by a least-square regression fit. The results of the statistical analysis for all channels are shown in detail in the validation report (Fennig et al.,
2017). An example for the channel at $37 \mathrm{GHz}$, h-pol is presented in Fig. 8.

Figure 8a shows $T_{\mathrm{B}}$ anomalies of the RDR without any modification or correction. Figure $8 \mathrm{~b}$ depicts the $T_{\mathrm{B}}$ anomalies after the corresponding corrections (reflector emissivity correction, sunlight intrusion, moonlight intrusion) and normalization (EIA normalization and diurnal cycle) are applied. A statistical model has been fitted to estimate the diurnal cycle in order to compare and check the homogenization of ascending and descending orbits (see the ATBD for more details; Fennig et al., 2017). Figure $8 \mathrm{c}$ shows the final $T_{\mathrm{B}}$ anomalies after the inter-calibration.

The time series of the raw data records show a very diverse picture. The agreement between the raw data records for SSM/I is generally better than for SSMIS. This is also evident for the homogenized data records that have been corrected for reflector emissivity, incidence angle, and diurnal cycle variations (second panel). Most $T_{\mathrm{B}}$ differences for the $\mathrm{SSM} / \mathrm{I}$ instruments are below $0.5 \mathrm{~K}$ in the RDR. The largest difference found for SSM/I is about $1 \mathrm{~K}$ in the $85 \mathrm{GHz}$ channels (see Figs. 11 and 12 in the SSM/I validation report). In contrast to this, most of SSMIS differences are between 0.5 and $1 \mathrm{~K}$. The ensemble spread between SSM/I and SSMIS (2006-2009) is larger than $3 \mathrm{~K}$ for the $37 \mathrm{GHz}$ channels. The best agreement between both sensor types is found for the $19 \mathrm{GHz}$ channels (see Figs. 6 and 7 in the SSMIS validation report) with about $1 \mathrm{~K}$ difference. This means that most of the observed inter-sensor differences before applying the inter-calibration offsets are larger than the estimated standard total uncertainty of the SSMI(S) instruments, which is about 0.7 to $1.1 \mathrm{~K}$ (see Sect. 6.2).

The calibration enhancement due to intrusion and reflector emissivity corrections (Fig. 8a, b) and the inter-calibration (Fig. 8b, c) reduce the seasonal variability and the intersensor variations and increase the quality and stability of the SSMI(S) data record significantly. The global climatological mean inter-satellite bias between SSM/I and SSMIS has been reduced to below $0.05 \mathrm{~K}$. As for the raw data record, the agreement in the final FCDRs between the individual SSM/I instruments is generally better than between the SSMIS instruments.

The FCDR significantly reduces the observed differences between the monthly means and shows very similar results for individual satellites. All global monthly mean intersensor differences are within $1 \mathrm{~K}$. Comparing the absolute maximum difference between the individual satellite biases to the ensemble mean, the CM SAF FCDR shows a remaining ensemble spread between 0.05 and $0.15 \mathrm{~K}$. No significant trend can be observed.

Overall, the monthly anomalies show a variability which is larger for horizontally than for vertically polarized channels and increases with higher frequencies. The increase in variability with higher frequency is mainly caused by the inclusion of all scenes; i.e. no rain filtering is applied. This additional noise can be interpreted as an additional uncertainty 
CM SAF FCDR :: TB h37 ensemble anomalies :: uncorrected data

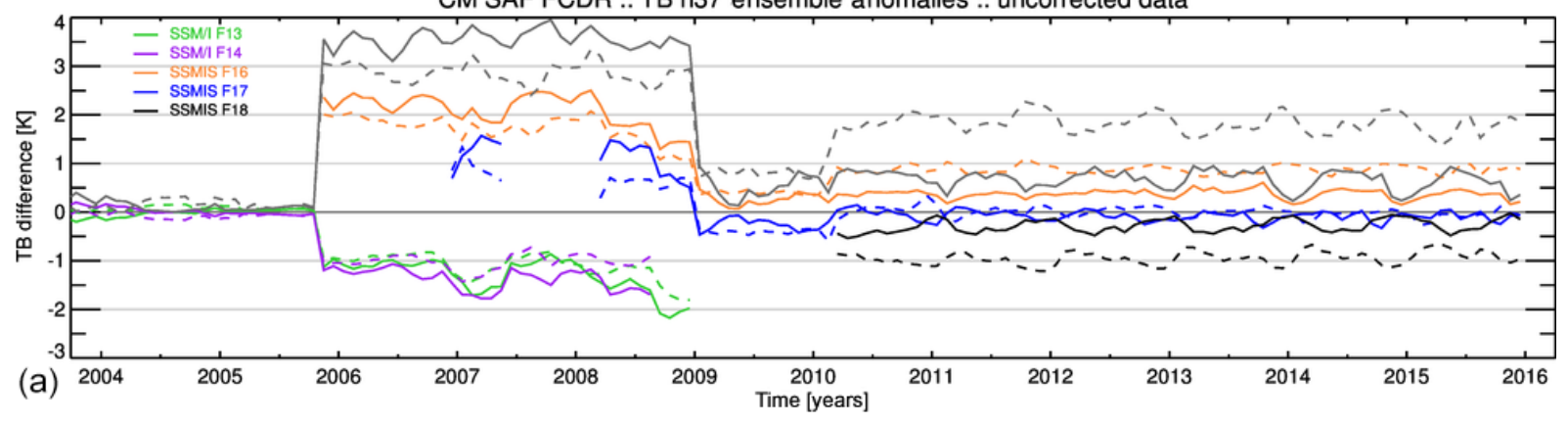

CM SAF FCDR :: TB h37 ensemble anomalies :: homogenized

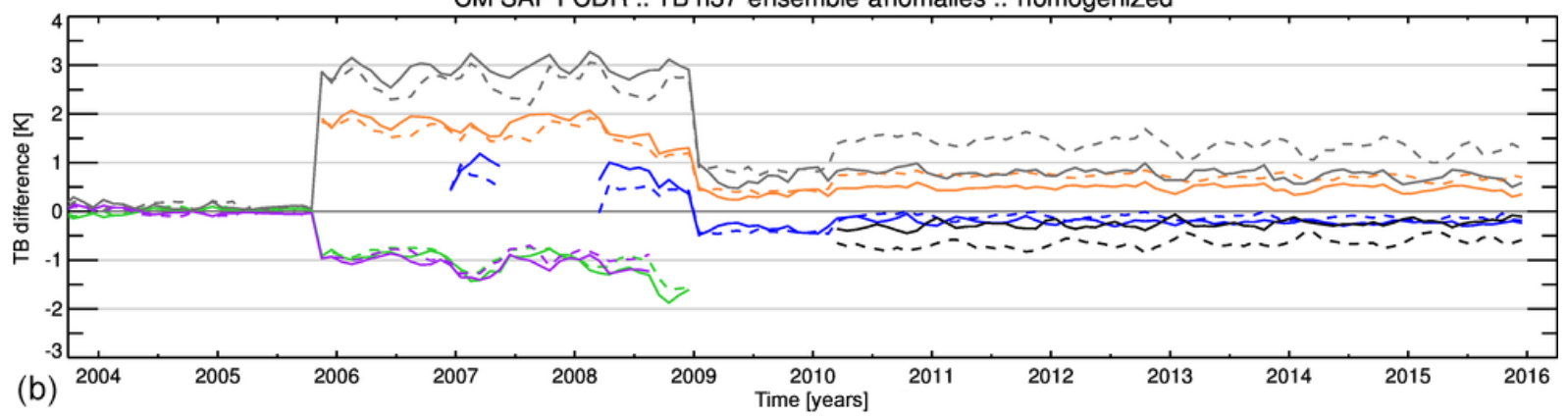

CM SAF FCDR :: TB h37 ensemble anomalies :: inter-calibration (daily mean)

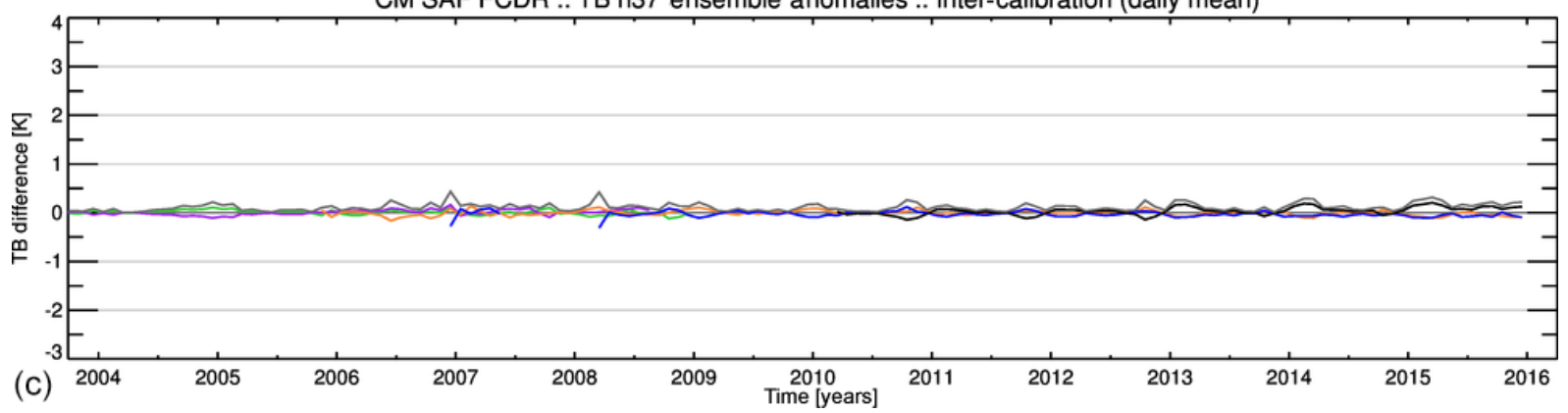

Figure 8. Time series of ensemble anomalies for SSM/I and SSMIS channels at $37 \mathrm{GHz}$, h-pol. In (a) and (b) the solid lines are PM orbits and the dashed lines AM orbits. Panel (c) shows daily means of AM and PM orbits. The grey lines depict the maximum difference observed between any two sensors (ensemble spread). For a detailed description see text. Colours are as follows: F16 - orange; F17 - blue; F18 black; F13 - green; F14 - purple.

due to differences in space and time collocation and sampling variability.

The robust standard deviations (see Figs. 6-12 in corresponding validation reports) depict constant values over time for all channels and satellites between 0.5 and $1 \mathrm{~K}$. This means that about $70 \%$ of all analysed monthly mean grid boxes are within \pm 0.5 to $\pm 1 \mathrm{~K}$.

Seasonal variations in the $T_{\mathrm{B}}$ differences at $91 \mathrm{GHz}$, caused by the reflector emissivity issue affecting the instruments on board F16 and F17, are of the order of $1 \mathrm{~K}$ for the global mean. These anomalies are corrected for the FCDR and only small variations remain in the homogenized plots (see Figs. 11 and 12 in the SSMIS validation report).

\subsection{Double differences for SMMR}

As mentioned before, an inter-sensor validation for SMMR was not conducted; instead double differences between observed and modelled $T_{\mathrm{B}}$ values are analysed. Ideally one would select one reference for the inter-calibration and another one for the evaluation. However, it was decided to use only ERA-20C (Poli et al., 2013) because it relies only on surface pressure and sea ice information and does not assimilate satellite data. Thus, ERA-20C is independent from the FCDR and also assumed to be more stable than ERA-Interim.

In order to minimize the influence of the diurnal cycle, unknown surface emissivities, and scattering effects, the comparison was limited to cloud-free scenes and scenes with small liquid water content over water surfaces. Brightness temperatures at the top of the atmosphere are calculated for 
the filtered data record as described in Sect. 5.3. In order to reduce the uncertainty in the observed differences, the matchup data are then gridded into daily global $1^{\circ}$ equal-angle grids, separately for ascending and descending orbits, and these are combined to daily means.

The monthly mean anomalies between the observed $T_{\mathrm{B}}$ and the modelled $T_{\mathrm{BM}}$ are calculated for each grid cell. Following the notation defined in the previous section, the anomalies at each grid point are then defined as

$\Delta T_{\mathrm{B}}(t, g, s)=T_{\mathrm{B}}(t, g, s)-T_{\mathrm{BM}}(t, g, s)$.

To compare the characteristics of the time series for both instruments, global median $\overline{\Delta T_{\mathrm{B}, s}}(t)$ and robust standard deviation $\operatorname{RSD}_{s}(t)$ are derived for each month.

Figure 9 shows the global monthly mean anomaly time series of SMMR and SSM/IF08 for the channels at $37 \mathrm{GHz}$ before and after inter-calibration. SSM/IF08 itself is intercalibrated to the SSM/I aboard F11, which is the FCDR calibration reference instrument. This means $\mathrm{SSM} / \mathrm{I}_{\mathrm{F} 08}$ is used as a transfer target. The relative differences without intercalibration range between -0.5 and $5.3 \mathrm{~K}$ for the channels at $37 \mathrm{GHz}$, h-pol and $37 \mathrm{GHz}$, v-pol respectively. Overall the SMMR anomalies depict a larger seasonal variation compared to SSM/I for the 19 and the $22 \mathrm{GHz}$ channel (not shown here). After applying the inter-calibration, all mean relative differences are significantly reduced to below $0.1 \mathrm{~K}$. The time series for the $37 \mathrm{GHz}$ channels are stable and a good continuity to $\mathrm{SSM} / \mathrm{I}_{\mathrm{F} 08}$ is apparent. The $21 \mathrm{GHz}$ channel depicts an upward trend of about $1 \mathrm{~K}$ from 1984 until it failed in March 1985 (not shown here). The $19 \mathrm{GHz}$ channels are showing a step in 1984 (not shown here). This corresponds to an observed EIA change in 1984 and might be an indication of unresolved issues with the attitude of the platform or some shortcoming in the surface emissivity model at that zenith angle. This is not evident in the $37 \mathrm{GHz}$ channels because the EIA dependence is smaller for higher frequencies. Also the seasonal variability is significantly larger for the lower frequencies, compared to SSM/I.

The anomalies are statistically analysed following the notations defined in the previous section. Recalling that about $70 \%$ of all data samples are within 1 standard deviation of a Gaussian distribution, the mean robust standard deviation is a statistical measure for the dispersion of all monthly mean $1^{\circ}$ anomalies. However, the natural variability and uncertainties in the forward model and the reanalysis data must also be accounted for. Therefore only the relative differences between SSM/IF08 and SMMR are important, not the absolute values. $\mathrm{SSM} / \mathrm{I}_{\mathrm{F} 08}$ is inter-calibrated to the reference instrument $\mathrm{SSM} / \mathrm{I}_{F 11}$, and the reanalysis is only used as a transfer standard. Under these conditions, all relative differences are within $0.1 \mathrm{~K}$ and are not statistically significant. The RSDs of the SMMR horizontally polarized channels are similar to those of SSM/I but the vertically polarized channels show larger RSDs. In particular the channel at $22 \mathrm{GHz}, \mathrm{v}-$ pol depicts a significantly larger noise (now shown).

\subsection{Comparison against reanalysis}

In addition to the inter-sensor comparison, a more complete analysis of each individual channel across the sensors for the complete time period was conducted. The idea is to derive a better characterization of the long-term FCDR stability, using independent reanalysis data records. However, this approach is hampered due to several constraints. The basic assumptions are that the reanalysis is independent of the observations, is stable in time, and does cover the complete time period of the FCDR. Moreover, the uncertainties in the applied radiative transfer model and the surface emissivity model should be well behaved and characterized. However, there are actually no reanalyses available that fulfil all these constraints.

The FCDR is compared to the ERA-20C reanalysis, similar to the SMMR inter-calibration and evaluation, as ERA20C does not assimilate any satellite observations. Note that the covered time period already ends in 2010. This does not allow a complete analysis of the transition from SSM/I to SSMIS with F18 data becoming available in 2010. Comparisons to the ERA-Interim reanalysis (Dee et al., 2011) would cover the full temporal coverage of the FCDR. However, ERAInterim assimilates SSM/I and SSMIS data records and is thus not independent. It was further shown that the usage of different SSM/I instruments leads to inhomogeneities in the analysed water vapour from ERA-Interim (e.g. Schröder et al., 2016). Thus, only results from the comparison to ERA20C are shown here.

A serious limitation in the comparison is the large uncertainty of surface emissivity models over land, ice, and snow-covered surfaces due to unknown surface emissivities. Also, the strong diurnal cycle over land is likely not fully resolved by the reanalysis time steps. Additionally, rainy and cloudy scenes increase the uncertainty of the radiative transfer model due to scattering effects of the water or ice droplets. Significant uncertainties are also caused by a lack of realistic cloud and rain profiles that feed the radiative transfer model, affecting all cloud and rain effects (emission, attenuation, and scattering). In order to minimize these influences, only cloud-free and lightly cloudy scenes (small liquid water content) over water surfaces are selected for the match-up data sets. However, this practically limits this comparison to the cold end of the natural spectrum, and no conclusion can be drawn for the scene dependence or the warm end of the spectrum.

Brightness temperatures are modelled using the same method as in the previous section, and the statistical analysis is also similar to the SMMR evaluation. The time series for AM and PM monthly mean anomalies and the RSD are shown exemplarily for the channel at $37 \mathrm{GHz}$, h-pol in Fig. 10. The figure contains three images with time series of monthly mean values of anomalies between RDRs (with reflector emissivity correction and intrusion correction applied) and ERA-20C (panel a), anomalies between inter-calibrated 


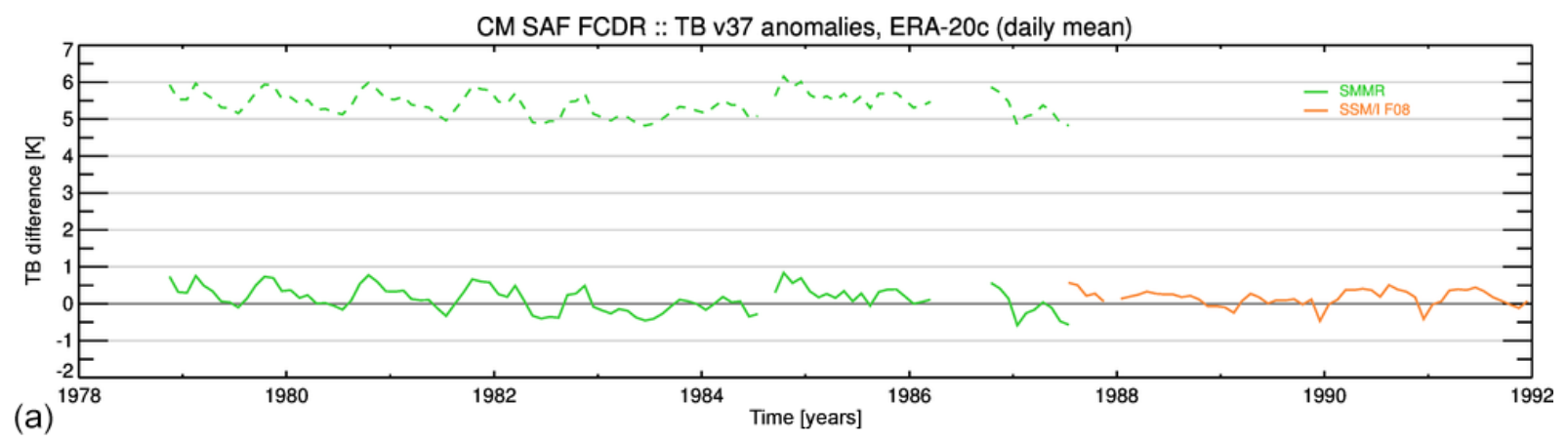

(a)

CM SAF FCDR :: TB h37 anomalies, ERA-20c (daily mean) 


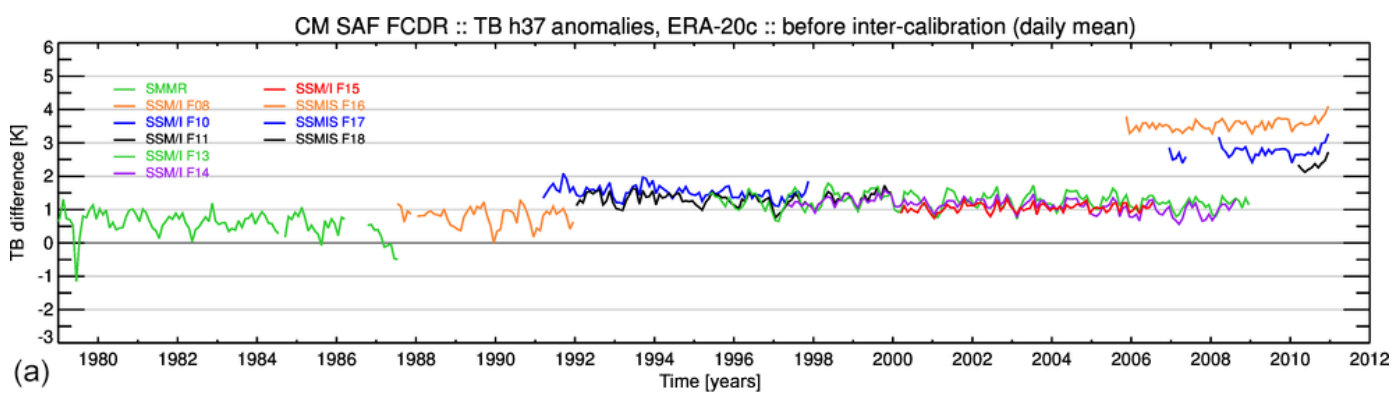

(a)

CM SAF FCDR :: TB h37 anomalies, ERA-20c :: with inter-calibration (daily mean)

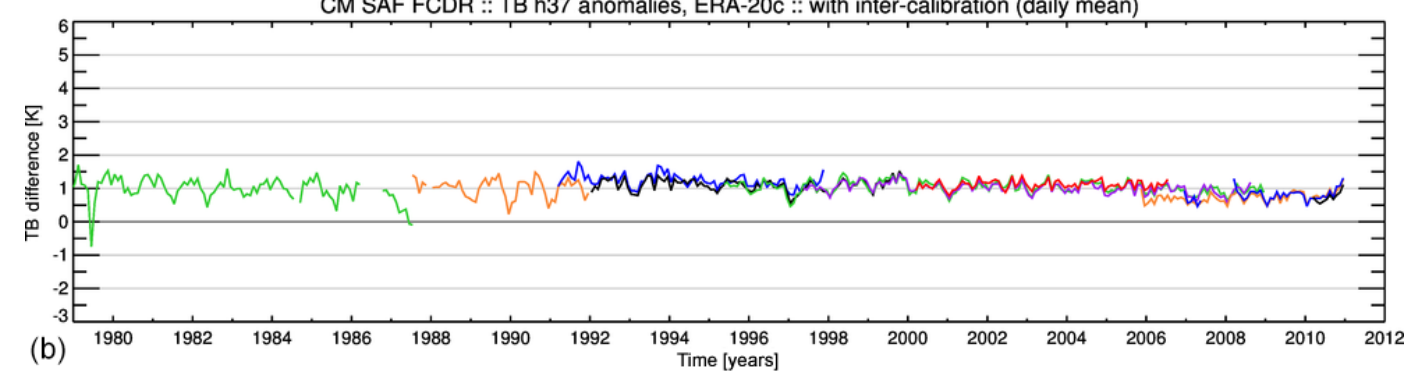

CM SAF FCDR :: TB h37 anomalies, ERA-20c :: robust standard deviation

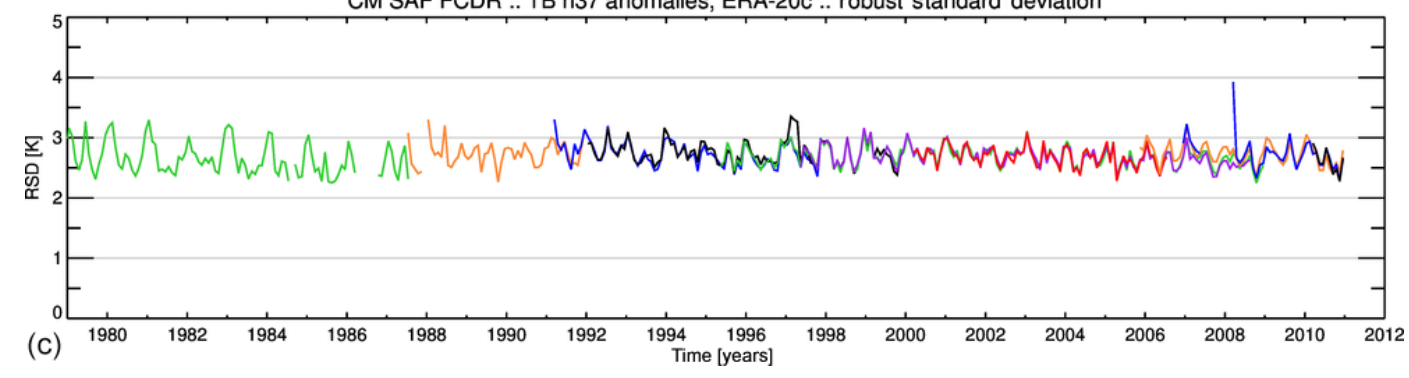

Figure 10. Time series of global monthly mean $T_{\mathrm{B}}$ differences for the $37 \mathrm{GHz}$, h-pol channel between the CM SAF FCDR and ERA-20C without inter-calibration (a) and with inter-calibration (b) and the RSD of the inter-calibrated data record and ERA-20C (c). Colours for the instruments are in parentheses: SMMR (green), F08 (orange), F10 (blue), F11 (black), F13 (green), F14 (violet), F15 (red), F16 (orange), F17 (blue), and F18 (black).

temperatures from radiometric cold scenes (rain-free ocean) to radiometric warm scenes (vegetated land surfaces). However, observations over warm land scenes can only be utilized in terms of polarization differences for the inter-sensor calibration procedure, in order to minimize the influence of the diurnal cycle. Therefore the uncertainty of the intercalibration is expected to be smaller over ocean than over land. The inter-calibration offsets are determined for all SSM/I-like channels. Other channels on SSMIS, not available on SSM/I, are also reprocessed, quality controlled, geolocated, and included in the FCDR data files. The consistency and homogeneity of the FCDR was statistically analysed to demonstrate the improvement of the reprocessed data records. The stability and homogeneity have also been tested by comparing the observed brightness temperatures against modelled brightness temperatures using reanalysis data.

The observed differences in the RDR range between 0.5 and $3 \mathrm{~K}$, depending on channel and instrument. Generally the differences are smaller within instruments from one series compared to the inter-sensor difference across the
SSMIS and SSM/I family. The overall mean differences in the CM SAF FCDR between the different sensors have been reduced to below $0.1 \mathrm{~K}$, which is a significant improvement over the RDR. The mean RSD for all channels and instruments has been significantly reduced. The observed residual variability in the inter-calibrated $T_{\mathrm{B}}$ values is mainly caused by the natural variability due to overpass time differences and sampling differences. No significant trend can be observed.

The SMMR has been evaluated using ERA-20C as a transfer standard. It was shown that the global mean anomalies of SMMR and SSM/I $\mathrm{F}_{08}$ agree well after inter-calibration. The SMMR channels depict higher values for the RSD than the corresponding SSM/I channels, but still at the same magnitude. This is mainly due to higher NEdT values and poorer coverage with a $50 \%$ duty cycle. In order to improve the quality of the SMMR data record and to better understand, quantify, and correct the remaining issues, it is essential to find and rescue the original level 1 data files.

Comparing against modelled brightness temperatures confirmed the results from the inter-sensor differences. However, 
it is still difficult to interpret the results in terms of temporal stability, as the reanalysis might also not be stable.

Finally it can be concluded that this FCDR is a fully documented and traceable data record providing greatly improved-quality SMMR and SSMI(S) brightness temperatures compared to original raw data records. It seamlessly extends the SSM/I data record with SSMIS data until the end of 2015 and continues it with SMMR data to 1978. The final combined FCDR thus provides inter-calibrated brightness temperatures for the time period from 1978 to 2015. The SSM/I part of this FCDR has already been used by the ERA5 reanalysis (Hersbach et al., 2018), and Lavergne et al. (2019) used the full FCDR for building a sea ice concentration data record.
It is planned to regularly update this FCDR within following continuous development and operations phases (CDOPs) of the CM SAF in order to extend the covered time period with recent SSMIS data and to further improve the quality by integrating user feedback. It is, amongst others, envisaged to improve the uncertainty characterization in future releases, work on the inter-calibration of the AMSR instrument family, and also look at new sensors like MWI and AMSR-3 for future usage. 
Appendix A: Computation of brightness temperatures

\section{A1 SSM/I}

For SSM/I, the variation in the polarization angle over the main lobe (FOV) can be neglected and the Earth antenna temperature equation is reduced to

$T_{\mathrm{A}_{p}}^{\prime}=\frac{1}{1-\Lambda_{\mathrm{sp}}} \int_{\Omega_{e}}\left[G_{p p} T_{\mathrm{B}_{p}}+G_{p q} T_{\mathrm{B}_{q}}\right] \mathrm{d} \Omega$.

Following Ashcroft and Wentz (2000), the cross-polarization leakage $\chi_{p}$ is defined as

$\chi_{p}=\frac{1}{1-\Lambda_{\mathrm{sp}}} \int_{\Omega_{e}} G_{p q} \mathrm{~d} \Omega$.

With the assumption that the general cross-polarization and co-polarization gain shapes are similar,

$G_{p q} \approx \frac{\chi_{p}}{1-\chi_{p}} \cdot G_{p p}$

this integral can be approximated as

$T^{\prime} \mathrm{A}_{p}=\frac{1}{1-\Lambda_{\mathrm{sp}}} \int_{\Omega_{e}}\left[G_{p p}\left(T_{\mathrm{B}_{p}}+\frac{\chi_{p}}{1-\chi_{p}} T_{\mathrm{B}_{q}}\right)\right] \mathrm{d} \Omega$.

Also, the antenna gains for vertical and horizontal polarization are nearly identical $G_{p p} \approx G_{q q}$. The integral over the co-polarization gain can be substituted as the mean $T_{\mathrm{B}}$ at polarization $p$ :

$\bar{T}_{\mathrm{B}_{p}}=\frac{1}{1-\chi_{p}} \cdot \frac{1}{1-\Lambda_{\mathrm{sp}}} \cdot \int_{\Omega_{e}} G_{p p} T_{\mathrm{B}_{p}} \mathrm{~d} \Omega$.

Equation (A4) can then be approximated as

$T^{\prime} \mathrm{A}_{v}=\left(1-\chi_{v}\right) \bar{T}_{\mathrm{B}_{v}}+\chi_{v} \bar{T}_{\mathrm{B}_{h}}$

$T^{\prime} \mathrm{A}_{h}=\left(1-\chi_{h}\right) \bar{T}_{\mathrm{B}_{h}}+\chi_{h} \bar{T}_{\mathrm{B}_{v}}$.

These two equations are solved for the scene $T_{\mathrm{B}}$, which yields

$\bar{T}_{\mathrm{B}_{v}}=T^{\prime} \mathrm{A}_{v}+\frac{\chi_{v}}{1-\chi_{v}-\chi_{h}} \cdot\left(T^{\prime} \mathrm{A}_{v}-T^{\prime} \mathrm{A}_{h}\right)$,

$\bar{T}_{\mathrm{B}_{h}}=T^{\prime} \mathrm{A}_{h}+\frac{\chi_{h}}{1-\chi_{v}-\chi_{h}} \cdot\left(T^{\prime} \mathrm{A}_{h}-T^{\prime} \mathrm{A}_{v}\right)$.

For the computation of the SSM/I $T_{\mathrm{B}}$ values, a fixed set of APCs is used, taken from Wentz (1991); instead of sensorspecific values. Colton and Poe (1999) concluded it would be best to use a fixed set of APCs for all sensors for a greater consistency across the sensors. The normalization of the polarization leakage factors as defined in Wentz (1991) $\left(\chi_{p}^{\prime}\right)$ is different compared to the definition in Eq. (A3), but can be converted by

$\chi_{p}=\frac{\chi_{p}^{\prime}}{1+\chi_{p}^{\prime}}$.

For the $22 \mathrm{GHz}$ channel no vertical polarization is measured, and Wentz (1991) fitted a linear function with a least-square regression to modelled oceanic $T_{\mathrm{B}}$ values and found

$\bar{T}_{\mathrm{B}_{v 22}}=1.01993 \cdot T_{\mathrm{A}_{v 22}}+1.994$.

The uncertainty of the approximation is about $0.1 \mathrm{~K}$. Another function was fitted for land observations. However, the differences to the expression over ocean are smaller than $0.2 \mathrm{~K}$ and only the equation above is applied.

\section{A2 SSMIS}

The SSMIS instrument has six individual feedhorns, which are placed at fixed angles from about -6 to $6^{\circ}$ along the focal plane of the reflector. This alignment leads to an additional polarization mixing, which has to be accounted for. The radiation entering each individual antenna feedhorn $T_{\mathrm{A}_{v, h}^{*}}$ is therefore a composition of the polarized radiation depending on the effective polarization angle $\gamma$ (similar to Eq. 19):

$T_{\mathrm{A}_{p}^{*}}=T_{\mathrm{A}_{p}} \cdot \cos ^{2} \gamma+T_{\mathrm{A}_{q}} \cdot \sin ^{2} \gamma$.

This set of equations can be solved to derive the antenna temperature aligned with the Earth polarization vector:

$T_{\mathrm{A}_{p}}=\frac{T_{\mathrm{A}_{p}^{*}} \cdot \cos ^{2} \gamma-T_{\mathrm{A}_{q}^{*}} \cdot \sin ^{2} \gamma}{\cos ^{2} \gamma-\sin ^{2} \gamma}$.

This rotation correction is applied to the SSM/I-like channels at 19, 37, and $91 \mathrm{GHz}$ before the antenna spillover (Eq. 21) and cross-polarization (Eq. A7) are corrected similar to SSM/I. For the sounding channels only a spillover correction is applied.

As for SSM/I, there is no horizontal polarization measured at $22 \mathrm{GHz}$. In this case a synthetic $T_{\mathrm{B}}$ is estimated from the $T_{\mathrm{B}}$ values at 19 and $22 \mathrm{GHz}$ from a least-square regression:

$\bar{T}_{\mathrm{B}_{h 22}}=c_{1}+c_{2} \bar{T}_{\mathrm{B}_{h 19}}+c_{3} \bar{T}_{\mathrm{B}_{v 19}}+c_{4} \bar{T}_{\mathrm{B}_{v 22}}$.

The values for coefficients $c$ are taken from the operational ground processing software configuration. Substituting this estimate into Eq. (A6) while accounting for the polarization rotation correction Eq. (A10) and defining

$\beta_{1}=\left(1-\chi_{v}\right) \cos ^{2} \gamma+\chi_{v} \sin ^{2} \gamma$,
$\beta_{2}=\left(1-\chi_{v}\right) \sin ^{2} \gamma+\chi_{v} \cos ^{2} \gamma$,

yields

$\bar{T}_{\mathrm{B}_{v 22}}=\frac{T^{\prime} \mathrm{A}_{v 22}-\beta_{2} \cdot\left(c_{1}+c_{2} \bar{T}_{\mathrm{B}_{h 19}}+c_{3} \bar{T}_{\mathrm{B}_{v 19}}\right)}{\beta_{1}+c_{4} \beta_{2}}$. 


\section{A3 SMMR}

In addition to the expected internal cross-polarization leakage at the instruments' polarization selector switches, high polarization mixing is induced as a result of the instrument design. The antenna system consists of a scanning parabolic reflector and a fixed multispectral feedhorn. Due to the antenna oscillating rotation, the alignment of the antenna pattern polarization vector relative to the Earth surface changes with increasing scan angle $\phi$. With increasing scan angle, vertical polarization is mixed into horizontal polarization and vice versa. Several studies (Gloersen et al., 1980; Gloersen, 1987; Francis, 1987) addressed this problem. The procedure used in the level 1B data records to correct for this effect was developed in Gloersen (1987) and implemented as follows.

The scan-angle-dependent integration of the antenna pattern over the antenna FOV can be derived similar to Eq. (A6) while keeping the antenna polarization vector dependence of the antenna gains:

$T_{\mathrm{A}_{p}}^{\prime}(\phi)=\left(1-\chi_{p}(\phi)\right) \cdot \bar{T}_{\mathrm{B}_{p}}+\chi_{p}(\phi) \cdot \bar{T}_{\mathrm{B}_{q}}$.

The antenna pattern cross-polarization leakage and switch polarization mixing effects are combined and approximated as follows (Njoku et al., 1998):

$\chi_{p}(\phi)=Q_{p} \cdot \sin ^{2}\left(\phi-\delta_{p}\right)$,

where $Q_{p}$ and $\delta_{p}$ are the amplitudes and phase shifts of the polarization mixing. The final set of coefficients is estimated empirically from in-orbit data and given in Njoku et al. (1998). Equation (A15) is then inverted to compute the scene $T_{\mathrm{B}}$ values from the normalized antenna temperatures:

$\bar{T}_{\mathrm{B}_{p}}=T^{\prime}{ }_{\mathrm{A}_{p}}+\frac{\chi_{p}(\phi)}{1-\chi_{q}(\phi)-\chi_{p}(\phi)} \cdot\left(T^{\prime}{ }_{\mathrm{A}_{p}}-T^{\prime}{ }_{\mathrm{A}_{q}}\right)$. 
Author contributions. All authors contributed to the generation of the data record and to the writing of the paper.

Competing interests. The authors declare that they have no conflict of interest.

Acknowledgements. This work was performed within the EUMETSAT CM SAF framework and we acknowledge the financial support of the EUMETSAT member states. The Nimbus-7 SMMR Pathfinder level 1B Brightness Temperature data record was provided by DAAC at the National Snow and Ice Data Center. The NOAA Climate Data Record of SSM/I and SSMIS Microwave Brightness Temperatures was provided by the National Centers for Environmental Information and the Colorado State University. The ERA-20C reanalysis was provided by ECMWF.

Review statement. This paper was edited by Ge Peng and reviewed by Thomas Lavergne and Catherine Prigent.

\section{References}

Andersson, A., Fennig, K., Klepp, C., Bakan, S., Graß1, H., and Schulz, J.: The Hamburg Ocean Atmosphere Parameters and Fluxes from Satellite Data - HOAPS-3, Earth Syst. Sci. Data, 2, 215-234, https://doi.org/10.5194/essd-2-215-2010, 2010.

Andersson, A., Klepp, C., Fennig, K., Bakan, S., Graß1, H., and Schulz, J.: Evaluation of HOAPS-3 ocean surface freshwater flux components, J. Appl. Meteor. Clim., 50, 379-398, https://doi.org/10.1175/2010JAMC2341.1, 2011.

Ashcroft, P. and Wentz, F. J.: Algorithm Theoretical Basis Document (ATBD), AMSR Level 2A Algorithm, Technical Report 121599B-1, Remote Sensing Systems, Santa Rosa, California, 2000.

Bell, W.: A Preprocessor for SSMIS Radiances, Scientific Description, Tech. Rep. NWPSAF-MO-UD-014, Version 1.0, 31.03.200, EUMETSAT NWP SAF, 2008.

Berg, W., Sapiano, M. R. P., Horsman, J., and Kummerow, C.: Improved Geolocation and Earth Incidence Angle Information for a Fundamental Climate Data Record of the SSM/I Sensors, IEEE T. Geosci. Remote, 51, 1504-1513, https://doi.org/10.1109/TGRS.2012.2199761, 2013.

Berg, W., Kroodsma, R., Kummerow, C. D., and McKague, D. S.: Fundamental Climate Data Records of Microwave Brightness Temperatures, Remote Sens., 10, 1306, https://doi.org/10.3390/rs10081306, 2018.

Colton, M. C. and Poe, G. A.: Intersensor Calibration of DMSP SSM/I's: F-8 to F-14, 1987-1997, IEEE T. Geosci. Remote, 37, 418-439, 1999.

Dee, D. P., Uppala, S., Simmons, A., Berrisford, P., Poli, P., Kobayashi, S., Andrae, U., Balmaseda, M., Balsamo, G., Bauer, P., Bechtold, P., Beljaars, A., van de Berg, L., Bidlot, J.-R., Bormann, N., Delsol, C., Dragani, R., Fuentes, M., Geer, A., Haimberger, L., Healy, S., Hersbach, H., Hólm, E., Isaksen, L., Kållberg, P., Köhler, M., Matricardi, M., McNally, A., Monge-Sanz, B., Morcrette, J.-J., Peubey, C., De Rosnay, P., Tavolato, C.,
Thepaut, J.-J., and Vitart, F.: The ERA-Interim reanalysis: Configuration and performance of the data assimilation system, Tech. rep., ECMWF, Shinfield Park, Reading, 2011.

Dorigo, W., Wagner, W., Albergel, C., Albrecht, F., Balsamo, G., Brocca, L., Chung, D., Ertl, M., Forkel, M., Gruber, A., Haas, E., Hamer, P. D., Hirschi, M., Ikonen, J., de Jeu, R., Kidd, R., Lahoz, W., Liu, Y. Y., Miralles, D., Mistelbauer, T., Nicolai-Shaw, N., Parinussa, R., Pratola, C., Reimer, C., van der Schalie, R., Seneviratne, S. I., Smolander, T., and Lecomte, P.: ESA CCI Soil Moisture for improved Earth system understanding: State-of-the art and future directions, Remote Sens. Environ., 203, 185-215, https://doi.org/10.1016/j.rse.2017.07.001, 2017.

Fennig, K., Andersson, A., and Schröder, M.: Fundamental Climate Data Record of SSM/I Brightness Temperatures, Dataset, Satellite Application Facility on Climate Monitoring, https://doi.org/ 10.5676/EUM_SAF_CM/FCDR_SSMI/V001, 2013.

Fennig, K., Andersson, A., and Schröder, M.: Fundamental Climate Data Record of SSM/I/SSMIS Brightness Temperatures, Dataset, Satellite Application Facility on Climate Monitoring, https://doi. org/10.5676/EUM_SAF_CM/FCDR_MWI/V002, 2015.

Fennig, K., Schröder, M., and Hollmann, R.: Fundamental Climate Data Record of Microwave Imager Radiances, Edition 3, Dataset, Satellite Application Facility on Climate Monitoring, https://doi. org/10.5676/EUM_SAF_CM/FCDR_MWI/V003, 2017.

Francis, E. A.: Calibration of the Nimbus-7 Scanning Multichannel Microwave Radiometer (SMMR) 1979-1984, Master's thesis, College of Oceanography, Oregon State University, Corvallis Oregon, 1987.

Fu, C. C., Han, D., Kim, S. T., and Gloersen, P.: User's Guide for the Nimbus-7 Scanning Multichannel Microwave Radiometer (SMMR) CELL-ALL Tape, NASA Reference Publication 1210, National Aeronautics and Space Administration, Washington, DC, 1988.

Furhop, R. and Simmer, C.: SSM/I Brightness Temperature Corrections for Incidence Angle Variations, J. Atmos. Ocean. Tech., 13, 246-254, 1996.

GCOS-200: GCOS Implementation Plan 2016, available at: https: //library.wmo.int/opac/doc_num.php?explnum_id=3417 (last access: 19 August 2019), 2017.

GLOBE Task Team: The Global Land One-kilometer Base Elevation (GLOBE) Digital Elevation Model, Version 1.0, Tech. rep, National Oceanic and Atmospheric Administration, National Geophysical Data Center, 325 Broadway, Boulder, CO, USA, available at: http://www.ngdc.noaa.gov/mgg/topo/globe. html (last access: 19 August 2019), 1999.

Gloersen, P.: In-Orbit Calibration Adjustment of the Nimbus-7 SMMR, NASA Technical Memorandum 100678, National Aeronautics and Space Administration, Washington, DC, 1987.

Gloersen, P. and Barath, F. T.: A Scanning Multichannel Microwave Radiometer for Nimbus-G and SeaSat-A, IEEE J. Oceanic Eng., OE-2, 271-278, 1977.

Gloersen, P., Cavalieri, D. J., and Soule, H. V.: An alternate Algorithm for Correction of the Scanning Multichannel Microwave Radiometer Polarization Radiances Using Nimbus-7 Observed Data, NASA Technical Memorandum 84976, Goddard Space Flight Center, Greenbelt, MD, 1980.

Hersbach, H., de Rosnay, P., Bell, B., Schepers, D., Simmons, A., Soci, C., Abdalla, S., Alonso-Balmaseda, M., Balsamo, G., Bechtold, P., Berrisford, P., Bidlot, J.-R., de Boisséson, 
E., Bonavita, M., Browne, P., Buizza, R., Dahlgren, P., Dee, D., Dragani, R., Diamantakis, M., Flemming, J., Forbes, R., Geer, A. J., Haiden, T., Hólm, E., Haimberger, L., Hogan, R., Horányi, A., Janiskova, M., Laloyaux, P., Lopez, P., MunozSabater, J., Peubey, C., Radu, R., Richardson, D., Thépaut, J.N., Vitart, F., Yang, X., Zsótér, E., and Zuo, H.: Operational global reanalysis: progress, future directions and synergies with NWP, ERA Report Series 27, ECMWF, Shinfield Park, Reading, https://doi.org/10.21957/tkic6g3wm, 2018.

Hollinger, J. P., Lo, R., Poe, G., Savage, R., and Peirce, J.: Special Sensor Microwave/Imager User's guide, NRL Technical Report, Naval Research Laboratory, Washington, DC, 1987.

Hollinger, J. P., Peirce, J. L., and Poe, G. A.: SSM/I Instrument Evaluation, IEEE T. Geosci. Remote, 28, 781-790, 1990.

Hollinger, J. P. et al.: DMSP Special Sensor Microwave/Imager Calibration/Validation I, NRL Technical Report, Naval Research Laboratory, Washington, DC, 1989.

Hoots, F. R. and Roehrich, L. R.: Models for Propagation of NORAD Element Sets, Spacetrack Report 3, available at: http:// celestrak.com/ (last access: 19 August 2019), 1988.

IERS: IERS Bulletins, available at: https://www.iers.org/IERS/ EN/Publications/Bulletins/bulletins.html, last access: 20 January 2020.

JCGM 100:2008: Evaluation of measurement data - Guide to the expression of uncertainty in measurement, Tech. rep, JCGM, 2008.

Kummerow, C. D., Berg, W. K., Sapiano, M. R. P., and Program, N. C.: NOAA Climate Data Record (CDR) of SSM/I and SSMIS Microwave Brightness Temperatures, CSU Version 1, Tech. rep., NOAA National Climatic Data Center, https://doi.org/10.7289/V5CC0XMJ, 2013.

Kunkee, D. B., Poe, G. A., Savage, R., and Peirce, J.: Special Sensor Microwave Imager Sounder (SSMIS) Radiometric Calibration Anomalies Part I: Identification and Characterization, IEEE T. Geosci. Remote, 46, 1017-1033, 2008a.

Kunkee, D. B., Poe, G. A., Swadley, S. D., Hong, Y., Wessel, J. E., and Uliana, E. A.: Desgin and Evaluation of the First Special Sensor Microwave Imager/Sounder, IEEE T. Geosci. Remote, 46, 863-883, 2008b.

Kunkee, D. B., Y., H., Thompson, D. A., Werner, M., and Poe, G. A.: Analysis of the Special Sensor Microwave Imager/Sounder (SSMIS) Fields-of-View on DMSP F-16, IEEE T. Geosci. Remote, 46, 934-944, 2008c.

Lavergne, T., Sørensen, A. M., Kern, S., Tonboe, R., Notz, D., Aaboe, S., Bell, L., Dybkjær, G., Eastwood, S., Gabarro, C., Heygster, G., Killie, M. A., Brandt Kreiner, M., Lavelle, J., Saldo, R., Sandven, S., and Pedersen, L. T.: Version 2 of the EUMETSAT OSI SAF and ESA CCI sea-ice concentration climate data records, The Cryosphere, 13, 49-78, https://doi.org/10.5194/tc-13-49-2019, 2019.

Madrid, C. R. et al.: The Nimbus 7 Users' Guide, NASA Technical Memorandum 79969, Goddard Space Flight Center, Greenbelt, MD, 1978.

Meunier, L. F., English, S., and Janssen, P.: Improved ocean emissivity modelling for assimilation of microwave imagers using foam coverage derived from a wave model, Tech. rep., EUMETSAT NWP SAF, 2014.

Njoku, E. G.: Nimbus-7 SMMR Pathfinder Brightness Temperatures, Version 1, Dataset, NASA National Snow and Ice Data Center Distributed Active Archive Center, https://doi.org/10.5067/7Y1XWXT07HH8, 2003.

Njoku, E. G., Rague, B., and Fleming, K.: The Nimbus-7 SMMR Pathfinder Brightness Temperature Data Set, JPL Publication 984, 1998.

Poe, G. A. and Conway, R. W.: A Study of the Geolocation Errors of the Special Sensor Microwave/Imager (SSM/I), IEEE T. Geosci. Remote, 28, 791-799, 1990.

Poe, G. A., Uliana, E. A., Gardiner, B. A., von Rentzell, T. E., and Kunkee, D. B.: Geolocation Error Analysis of the Special Sensor Microwave Imager/Sounder, IEEE T. Geosci. Remote, 46, 913922, 2008.

Poli, P., Dee, D., Saunders, R., John, V., Rayer, P., Schulz, J., Holmlund, K., Coppens, D., Klaes, D., Johnson, J., Esfandiari, A., Gerasimov, I., Zamkoff, E., Al-Jazrawi, A., Santek, D., Albani, M., Brunel, P., Fennig, K., Schröder, M., Kobayashi, S., Oertel, D., Döhler, W., Spänkuch, D., and Bojinski, S.: Recent advances in satellite data rescue, B. Am. Meteorol. Soc., 98, 1471-1484, https://doi.org/10.1175/BAMS-D-15-00194.1, 2017.

Poli, P., Hersbach, H., Tan, D. G. H., Dee, D. P., Thépaut, J-N., Simmons, A., Peubey, C., Laloyaux, P., Komori, T., Berrisford, P., Dragani, R., Trémolet, Y., Hólm, E. V., Bonavita, M., Isaksen, L., and Fisher, M.: The data assimilation system and initial performance evaluation of the ECMWF pilot reanalysis of the 20th-century assimilating surface observations only (ERA-20C), ERA Report Series 14, ECMWF, Shinfield Park, Reading, 2013.

Prigent, C., Jimenez, C., and Aires, F.: Toward "all weather", long record, and real-time land surface temperature retrievals from microwave satellite observations, J. Geophys. Res.-Atmos., 121, 5699-5717, https://doi.org/10.1002/2015JD024402, 2016.

Ritchie, A. A., Smith, M. R., Goodman, H. M., Schudalla, R. L., Conway, D. K., LaFontaine, F. J., Moss, D., and Motta, B.: Critical Analyses of Data Differences Between FNMOC and AFGWC Spawned SSM/I Datasets, J. Atmos. Sci., 55, 16011612, 1998.

Rivest, R.: The MD5 Message-Digest Algorithm, Tech. rep., https://doi.org/10.17487/RFC1321, 1992.

Sapiano, M. R. P., Berg, W. K., McKague, D. S., and Kummerow, C. D.: Toward an Intercalibrated Fundamental Climate Data Record of the SSM/I Sensors, IEEE T. Geosci. Remote, 51, 1492-1503, https://doi.org/10.1109/TGRS.2012.2206601, 2013.

Saunders, R., Hocking, J., Rundle, D., Rayer, P., Matricardi, M., Geer, A., Lupu, C., Brunel, P., and Vidot, J.: RTTOV-11 science and validation report, Tech. rep., EUMETSAT NWP SAF, available at: http://nwpsaf.eu/deliverables/rtm/rtm_rttov11.html (last access: 19 August 2019), 2013.

Schröder, M., Lockhoff, M., Forsythe, J., Cronk, H., Haar, T. H. V., and Bennartz, R.: The GEWEX water vapor assessment (GVAP) - results from the trend and homogeneity analysis, J. Appl. Meteor. Clim., 55, 1633-1649, https://doi.org/10.1175/JAMCD-15-0304.1, 2016.

Schulz, J., Albert, P., Behr, H.-D., Caprion, D., Deneke, H., Dewitte, S., Dürr, B., Fuchs, P., Gratzki, A., Hechler, P., Hollmann, R., Johnston, S., Karlsson, K.-G., Manninen, T., Müller, R., Reuter, M., Riihelä, A., Roebeling, R., Selbach, N., Tetzlaff, A., Thomas, W., Werscheck, M., Wolters, E., and Zelenka, A.: Operational climate monitoring from space: the EUMETSAT Satellite Application Facility on Climate Monitoring (CM-SAF), Atmos. Chem. 
Phys., 9, 1687-1709, https://doi.org/10.5194/acp-9-1687-2009, 2009.

Semunegus, H., Berg, W., Bates, J. J., Knapp, K. R., and Kummerow, C.: An Extended and Improved Special Sensor Microwave Imager (SSM/I) Period of Record, J. Climate Appl. Meteor., 49, 424-436, 2010.

Stogryn, A.: Estimates of brightness temperatures from scanning radiometer data, IEEE T. Geosci. Remote, 26, 720-726, https://doi.org/10.1109/TAP.1978.1141919, 1978.

Swift, C. T., Fedor, L. S., and Ramseier, R. O.: An Algorithm to Measure Sea Ice Concentration with Microwave Radiometers, J. Geophys. Res.-Oceans, 90, 1087-1099, 1985.
Wentz, F. J.: User's Manual for SSM/I Antenna Temperature Tapes Revision 1, Technical Report 120191, Remote Sensing Systems, Santa Rosa, CA, USA, 1991.

Wentz, F. J., Mears, C. A. M., and Program, N. C.: NOAA Climate Data Record (CDR) of SSM/I and SSMIS Microwave Brightness Temperatures, RSS Version 7, Tech. rep., NOAA National Centers for Environmental Information, https://doi.org/10.7289/V5SJ1HKZ, 2013. 